\title{
Adaptive and Sliding Mode Friction-Resilient Machine Tool Positioning - Cascaded Control Revisited
}

Papageorgiou, Dimitrios; Blanke, Mogens; Niemann, Hans Henrik; Richter, Jan H.

\section{Published in:}

Mechanical Systems and Signal Processing

Link to article, DOI:

10.1016/j.ymssp.2019.06.015

Publication date:

2019

Document Version

Early version, also known as pre-print

Link back to DTU Orbit

Citation (APA):

Papageorgiou, D., Blanke, M., Niemann, H. H., \& Richter, J. H. (2019). Adaptive and Sliding Mode FrictionResilient Machine Tool Positioning - Cascaded Control Revisited. Mechanical Systems and Signal Processing, 132, 35-54. https://doi.org/10.1016/j.ymssp.2019.06.015

\section{General rights}

Copyright and moral rights for the publications made accessible in the public portal are retained by the authors and/or other copyright owners and it is a condition of accessing publications that users recognise and abide by the legal requirements associated with these rights.

- Users may download and print one copy of any publication from the public portal for the purpose of private study or research.

- You may not further distribute the material or use it for any profit-making activity or commercial gain

- You may freely distribute the URL identifying the publication in the public portal 


\title{
Adaptive and Sliding Mode Friction-Resilient Machine Tool Positioning - Cascaded Control Revisited
}

\author{
Dimitrios Papageorgiou ${ }^{\mathrm{a}}$, Mogens Blanke ${ }^{\mathrm{a}, \mathrm{b}}$, Hans Henrik Niemann ${ }^{\mathrm{a}}$, Jan H. Richter ${ }^{\mathrm{c}}$ \\ ${ }^{a}$ Department of Electrical Engineering, Automation and Control, Technical University of Denmark, DK-2800 Kgs. Lyngby, Denmark, \\ dimpa,mb,hhn@elektro.dtu.dk \\ ${ }^{b}$ AMOS CoE, Institute of Technical Cybernetics, Norwegian University of Science and Technology, Trondheim, Norway \\ ${ }^{c}$ Siemens AG, Digital Factory Division, D-90475 Nuremberg, Germany janrichter@ siemens.com
}

\begin{abstract}
Needs for high-accuracy tool positioning and accurate trajectory following have renewed the focus on controller design for machine tools. While state-of-the-art solutions, based on Proportional (P) and Proportional-Integral (PI) cascades, achieve sufficient nominal performance, axis positioning accuracy quickly degrades in the presence of additional wear-related friction. Sliding-mode and nonlinear adaptive controllers with no cascaded architecture can alleviate such performance deterioration at the cost, however, of significantly increased design complexity. This is mainly due to the fact that such architectures facilitate addressing more nonlinear phenomena, such as load dynamic friction. This paper investigates three nonlinear controllers with cascaded architecture for machine tool axis positioning. A comparative analysis of the positioning solutions is carried out and it is shown that a cascaded scheme comprising a proportional and a super-twisting sliding-mode controller offers superior friction-resilient axis positioning. Moreover, its design complexity is comparable to that of the conventional P-PI solution. Experimental results obtained from a single-axis test setup equipped with commercial industrial equipment validate the theoretical findings.
\end{abstract}

Keywords: Machine-tool control, axis drive-train, friction, super-twisting sliding-mode control, nonlinear adaptive control, immersion and invariance control, robustness, high accuracy positioning.

\section{Introduction}

Modern industrial manufacturing using automated machine tools requires high-accuracy positioning of the machine axes. A number of mechanical phenomena, especially friction, may compromise the appropriate positioning of the machine endtool relatively to the workpiece, thus degrading the finishing quality. To ensure that workpiece tolerances are maintained, the machine drives are equipped with position-control algorithms that take into account the friction characteristics of the axes.

State-of-the-art machine tool axis control solutions comprise cascades of P and PI controllers for the axis position and velocity, respectively. The position controller outputs a velocity reference to the velocity controller, which in turn, delivers the appropriate torque command for the motor that will move the axis to the desired position [1]. A large corpus of documented machine tool positioning methods exists in the literature (see $[2,3,4,5]$ and the references therein for a general overview and classification of the methods). Variable-gain P-PI approaches have also been made for improving accuracy in motion control systems [6,7] and transient performance [8].

The effect of friction on machine axis positioning is usually counteracted by including appropriate feedforward terms in the P-PI control scheme $[9,10]$. The structure and complexity of the compensating solutions is related to the level of detail at which the various friction phenomena are described [11], as well as the regime of the axis motion. Modelling and com- pensation of friction during slow axis motion was pursued in $[12,13]$. Presliding friction was addressed in [14] for a twomode motion profile. Observers were used in [15], in which friction was considered a disturbance signal. An approach for friction estimation based on model inversion was made in $[16,17]$. A comparative study on model-based and model-free methods for compensating friction via feed-forwards terms was presented in [18]. A feedforward observer was used in [19, 20] for compensation of nonlinear presliding friction. The design of this observer, tailored for motion control applications, was generalized in [21]. The combination of cascaded P-PI motion controls with nonlinear friction rejection at micro-positioning was evaluated in $[22,23]$, where the authors demonstrated the methods' performance limitations in terms of steady-state positioning accuracy. Feed-forward compensation of friction was pursued in [24], where a frequency domain characterisation of the nonlinear friction was obtained, using low amplitude/low frequency excitation signals. Particle Swarm Optimizationbased tuning of a PID controller was used in [25] to achieve sufficient compensation of nonlinenar friction.

The majority of these compensation schemes assume constant friction model parameters, which are obtained from offline identification experiments [26, 27]. Under nominal conditions these conventional axis positioning solutions achieve sufficient accuracy. However, they quickly degrade in performance if the friction parameters change. The need for friction-resilient operation even under equipment wear has motivated research in 
the area of model-based nonlinear control for friction compensation.

A substantial amount of work on nonlinear adaptive and Sliding Mode Control (SMC) methods has been reported in the literature in relation to friction rejection. The reader is indicatively referred to $[28,29,30,31,32,33]$, as well as to $[34,35,36,37,38,39,40,41]$. Although the powerful robustness properties of these controller families have been extensively analysed, their performance and applicability to machine tool control had not been adequately assessed, especially in comparison to the conventional P-PI cascades. Such a comparative study was carried out in [42], in which the performance of three nonlinear controllers, namely of an Adaptive Backstepping Controller (ABSC), an Output Super-twisting Sliding Mode Controller (OSTSMC) and an Adaptive Super-twisting Sliding Mode Controller (ASTSMC), was experimentally assessed with respect to high-accuracy and robustness against unknown and increasing friction. All three controllers shared the same direct-position architecture, i.e. no explicit positionvelocity cascades were included in their design. The results showed that the ABSC offered significantly higher accuracy in axis positioning even under conditions of unknown friction. This however, came at the cost of increased complexity, since the design of the controller required the tuning of 19 parameters.

This paper extends the study done in [42] by revisiting the position-velocity cascaded architecture for machine tool axis positioning. The proposed designs comprise a $\mathrm{P}$ controller for the position loop, followed by a nonlinear controller for the motor velocity. The motivation for this approach was to utilize the friction-resilience of the nonlinear controllers and at the same time reduce the design complexity that direct positioning methods introduce. Similarly to [42], the designs are based on sliding-mode and nonlinear-adaptive control principles. Specifically, a Super-twisting Sliding Mode Controller (STSMC), a certainty-equivalence Nonlinear Adaptive Controller (NAC) and a Immersion and Invariance Adaptive Controller (I\&I-AC) are proposed for the motor velocity. The theoretical properties of each solution are elaborated and the controller's performance is assessed through experiments conducted on state-of-the-art machine tool equipment. The proposed nonlinear methods are systematically compared to the standard P-PI cascade and to the controllers from [42], based on criteria for robust positioning accuracy, efficiency and overall complexity of each method.

The paper is structured as follows: Section 2 describes the considered physical system and presents the problem being addressed, while Section 3 introduces the mathematical model of the machine axis. Section 4 presents the design of the proposed control schemes used for axis positioning, along with the stability and convergence properties of the closed-loop system. Experimental results on the performance of the control schemes are presented in Section 5, while Section 6 offers an overall comparison of the controllers to the methods proposed in [42]. Finally, conclusions and future work are discussed in Section 7.

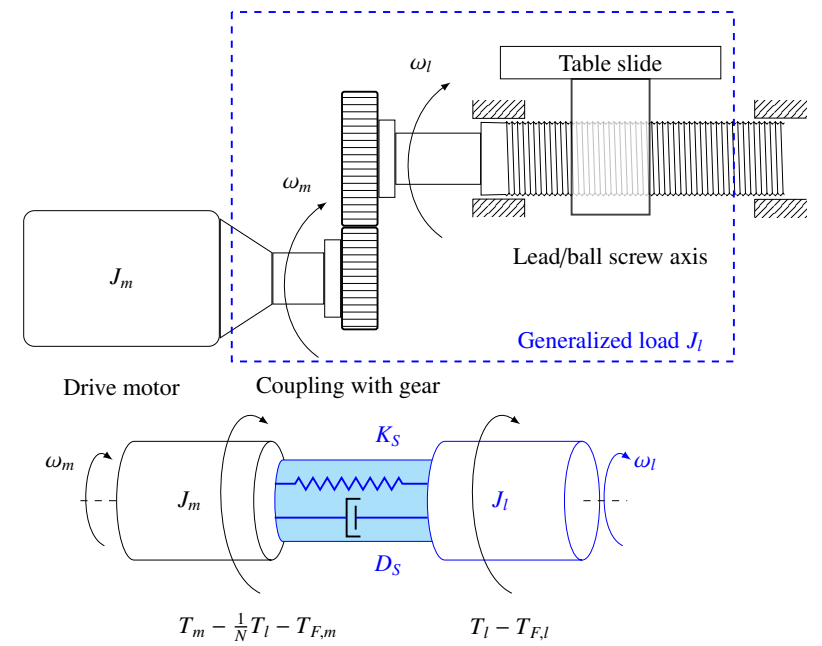

Figure 1: (Top) Machine-tool axis, where $\omega_{m}, \omega_{l}$ are the motor and load angular velocities, respectively. (Bottom) Equivalent double mass oscillator with $J_{m}, J_{l}$ being the drive motor and generalized load inertias, respectively, $K_{S}$ the spring constant corresponding to the shaft stiffness and $D_{S}$ being the damping coefficient of the shaft. The torque generated by the drive motor is denoted with $T_{m}$, the interconnecting torque is $T_{l}, N=1$ is the gearing ratio between motor and load and $T_{F, m}, T_{F, l}$ is the friction on the motor and the load side, respectively.

\section{System description and problem formulation}

\subsection{System in context and accuracy}

A machine tool axis can be considered as a system of two interacting inertias (motor and load) connected through a shaft with friction. The inertia and friction on the load side corresponds to the lumped masses and friction torques on the linear axis of the machine, as illustrated in Figure 1. The drive-train abstraction introduced in [42] for describing a machine tool axis is also adopted in this paper.

The purpose of a machine tool axis control is to achieve accurate tracking of a position profile by the axis end-point (e.g. the tool), i.e. to accurately position the generalized load. In standard machine tool applications the required workpiece tolerances correspond to a positioning accuracy in the range $1-10 \mu \mathrm{m}$. For machines with linear axes that typically have lead screws with pitch size $2-10 \mathrm{~mm}$, such requirements correspond to an angular positioning accuracy between $5 \cdot 10^{-3}$ and $2 \cdot 10^{-2} \mathrm{rad}[1]$. The accuracy requirement considered in this study is $10^{-2} \mathrm{rad}$.

\subsection{Positioning-degrading friction}

The decelerating friction forces that develop between the various surfaces of the machine parts may hinder the accurate positioning of the axis. Although the effects of friction are usually accounted for when commissioning the machine drive, these feed-forward compensation schemes assume constant friction characteristics. However, these characteristics can change over time due to equipment wear and varying environmental conditions. Typical examples include deformation of the bearing surfaces of the motors and linear axes, lubrication film failure or low room temperature, which can cause increased Coulomb friction and viscosity [43]. 
The friction acting on the drive motor is different than the one affecting the load. The first is primarily due to the contact of the bearing surfaces and possibly additional heat losses due to eddy currents. On the other hand, load friction develops in the gearing mechanism, the linear axis ball screw system and the various intermediate contact surfaces. However, since the cascaded control architectures discussed in this study concern only the drive motor dynamics, the load friction is not explicitly modelled. Especially in the case of stiff machine axes, changes in load friction are directly reflected in the motor friction parameters.

\subsection{Problem formulation}

The control objective of this study is stated in the following:

Problem 1 (Friction-resilient accuracy control for single-axis machine tool). Consider a single-axis machine tool system consisting of a drive motor connected to a load with a flexible shaft. Let the tool positioning error be denoted by $e_{\theta}$ and let $T_{C, m}^{\max }$ be an upper bound for the Coulomb friction magnitude $T_{C, m}$ on the motor side. Design a closed-loop control strategy that ensures:

$$
\left|e_{\theta}(t)\right| \leq 10 \operatorname{mrad} \forall t \geq t_{0}>0 \text { and for } T_{C, m} \leq T_{C, m}^{\max }
$$

where $t_{0}$ denotes a time after the starting up of the positioning task for the machine.

In the above problem formulation the bound $T_{C, m}^{\max }$ describes the maximum value of Coulomb friction, above which alleviation of the positioning degradation is not addressed by means of low-level axis control.

\section{Mathematical model}

The drive-train system consists of the drive motor, the shaft and the load. The closed-loop electrical dynamics of the motor can be approximated by a first-order system with time constant much faster than the mechanical dynamics of the single-axis system. It is, therefore, a common assumption in motion control studies that the torque produced by the motor is the torque command plus a torque disturbance $d_{e}$, i.e. $T_{m}=u+d_{e}$ as shown in figure 2 .

Apart from the fast electrical dynamics, the torque perturbation $d_{e}$ includes several cogging torques and parasitic ripples coming from current sensor offsets and inverter nonlinearities. Analytical modelling and identification methods of such torque ripples have been reported in the literature [44, 45, 46, 47, 48], while compensation of the input torque perturbation is mainly achieved via appropriate feed-forward terms in the current control loop [49, 50] and it is not addressed in the current study. Instead, the assumption that there exists appropriate feedforward compensation of $d_{e}$, introduced in [42], is also adopted here, i.e.

$$
T_{m} \approx u
$$

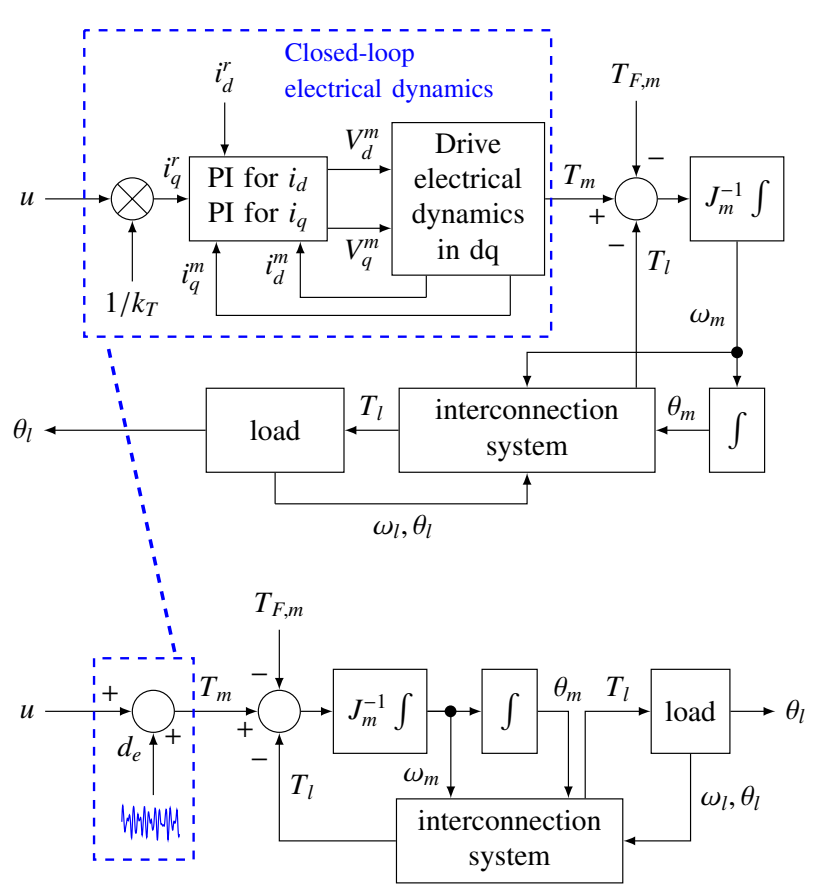

Figure 2: (Top) Single axis system with closed-loop electrical dynamics. The torque command $u$ is translated into the quadrature current reference signal $i_{q}^{r}$ through the motor torque constant $k_{T}$. (Bottom) Reduced-order single-axis system.

The dynamics of the drive-train read:

$$
\begin{aligned}
\dot{\omega}_{m} & =\frac{1}{J_{m}} u-\frac{1}{J_{m}} T_{F, m}-\frac{1}{N J_{m}} T_{l} \\
\dot{\theta}_{m} & =\omega_{m} \\
\dot{\omega}_{l} & =-\frac{1}{J_{l}} T_{F, l}+\frac{1}{J_{l}} T_{l} \\
\dot{\theta}_{l} & =\omega_{l}
\end{aligned}
$$

where $T_{F, l}$ is the friction on the load side and the interconnecting torque $T_{l}$ is given from

$$
T_{l}=K_{S}\left(\frac{1}{N} \theta_{m}-\theta_{l}\right)+D_{S}\left(\frac{1}{N} \omega_{m}-\omega_{l}\right) .
$$

The friction torque $T_{F, m}$ acting on the motor is described by the following equation [51]:

$$
T_{F, m}=\left[T_{C, m}+\left(T_{S, m}-T_{C, i}\right) e^{-\left(\frac{\omega_{m}}{\omega_{S}}\right)^{2}}\right] \operatorname{sgn}\left(\omega_{m}\right)+\beta_{m} \omega_{m}
$$

where the Stribeck velocity $\omega_{S}$ is considered known from offline identification and $\operatorname{sgn}(\cdot)$ is the signum function defined as

$$
\operatorname{sgn}(y)= \begin{cases}1 & \text { if } y>0 \\ v \in[-1,1] & \text { if } y=0 \\ -1 & \text { if } y<0\end{cases}
$$

Apart from the Coulomb and viscous components, this friction model also includes the stiction aspect that relates to the presliding phenomenon [52]. During very slow axis motion profiles (almost zero speed) the stick-slip phenomenon is the 


\begin{tabular}{|c|c|c|}
\hline Symbol & $\begin{array}{l}\text { Table 1: System model nomenclature. } \\
\text { Description }\end{array}$ & Units \\
\hline \multicolumn{3}{|c|}{ States and Outputs } \\
\hline$\omega_{m}, \omega_{l}$ & Motor/load angular velocity & $\mathrm{rad} \mathrm{s}^{-1}$ \\
\hline$\theta_{m}, \theta_{l}$ & Motor/load angular position & $\mathrm{rad}$ \\
\hline \multicolumn{3}{|l|}{ Inputs } \\
\hline$\theta_{r}$ & Load position reference & $\mathrm{rad}$ \\
\hline$\omega_{r}$ & Motor velocity reference & $\operatorname{rad~s}^{-1}$ \\
\hline$u$ & Torque command & $\mathrm{Nm}$ \\
\hline \multicolumn{3}{|c|}{ Constant parameters } \\
\hline$N$ & Gearing ratio & - \\
\hline$J_{m}, J_{l}$ & Motor/load inertia & $\mathrm{kg} \mathrm{m}^{2}$ \\
\hline$K_{S}$ & Shaft stiffness & $\mathrm{N} \mathrm{m} \mathrm{rad}{ }^{-1}$ \\
\hline$D_{S}$ & Shaft damping coefficient & $\mathrm{Nmsrad}-1$ \\
\hline$T_{C, m}$ & Motor Coulomb friction & $\mathrm{Nm}$ \\
\hline$T_{S, m}$ & Motor static friction coefficient & $\mathrm{Nm}$ \\
\hline$\omega_{S}$ & Motor Stribeck velocity & $\operatorname{rad~s}^{-1}$ \\
\hline$\beta_{m}$ & Motor viscous friction coefficient & $\mathrm{Nms} \mathrm{rad}{ }^{-1}$ \\
\hline \multicolumn{3}{|c|}{ Disturbances } \\
\hline$d_{e}$ & Input torque ripples and harmonics & $\mathrm{Nm}$ \\
\hline$T_{F, m}, T_{F, l}$ & Motor/load friction & $\mathrm{Nm}$ \\
\hline$T_{l}$ & Load torque & $\mathrm{Nm}$ \\
\hline
\end{tabular}

most dominant friction component. More sophisticated descriptions of friction have been proposed for such regimes in [53, 54, 27, 55, 56, 57]. However, the complexity introduced by these models, significantly increases the difficulty of designing, implementing and maintaining nonlinear model-based solutions for friction compensation. This is mainly due to additional dynamics and large number of unknown parameters (see for example the LuGre [58] and Generalized Maxwell Slip [59] friction models). At low speeds the "limit" behaviour of friction, reflected in the stiction coefficient (or breakaway torque) is sufficient to facilitate effective friction compensation. A list with the most important variables and notation of the drive train model is provided in Table 1.

\section{Axis position control methods}

\subsection{Control architecture}

The advanced nonlinear control strategies to position the load will be discussed in this section. Three different control algorithms based on sliding-mode and nonlinear adaptive principles are proposed for robust axis positioning with respect to unknown and increasing Coulomb friction. All three designs have a position-velocity cascaded architecture. This structure maintains the outer loop P controller of the P-PI cascade in standard machine tool control, but the velocity PI controller is replaced by its nonlinear counterpart, as shown in Figure 3. This implies that all the proposed designs start from considering the drive motor velocity error dynamics.

Similar to the conventional P-PI cascades, the motor velocity reference $\omega_{r}$ is the output of the position proportional controller plus a feedforward term that is equal to the first derivative of the position reference $\dot{\theta}_{r}$, i.e.

$$
\omega_{r}=k_{p o s} e_{\theta}+N \dot{\theta}_{r}
$$

where $e_{\theta} \triangleq \theta_{r}-\theta_{l}$ is the axis positioning error and $k_{p o s}>0$ is the gain of the $\mathrm{P}$ controller. Since the time derivatives of the reference signal are usually available up to the acceleration level, the derivative of the velocity reference $\omega_{r}$ can also be calculated by differentiating (4.1), as shown below:

$$
\dot{\omega}_{r}=\frac{d}{d t}\left(k_{p o s} e_{\theta}+N \dot{\theta}_{r}\right)=k_{p o s}\left(\dot{\theta}_{r}-\omega_{l}\right)+N \ddot{\theta}_{r} .
$$

If $e_{\omega} \triangleq \omega_{m}-\omega_{r}$ is the motor velocity tracking error, then it is easy to show that the position error dynamics are given by

$$
\dot{e}_{\theta}=-\frac{k_{p o s}}{N} e_{\theta}-\frac{1}{N} e_{\omega}+\Delta \omega
$$

with $\Delta \omega \triangleq \frac{1}{N} \omega_{m}-\omega_{l}$ being the velocity difference between motor and load. The magnitude of this term is negligible for rigid connection between motor and load, while in case of flexible shafts it plays a key role in selecting the proportional gain $k_{p o s}$, together with the motor-load position difference and the shaft compliance $[60,61]$. From (4.3) it can be seen that after some transients, it holds that $\left|e_{\theta}\right| \leq \frac{1}{k_{p o s}}\left(\left|e_{\omega}\right|+N|\Delta \omega|\right)$, i.e. appropriate bounds on the velocity error give sufficient positioning accuracy.

Compared to solutions employing direct load position nonlinear controllers [42], this architecture facilitates relatively simple designs (the controlled system is scalar) and offers some degree of modularity, since the position and velocity controllers can be tuned separately. The low design complexity allows for enriching the friction description with stiction, as mentioned in Section 3. This inclusion was motivated from the fact that stiction contributes to positioning degradation during axis motion reversals. As it will become clear in Section 4, this modelling choice only affects the design of the proposed adaptive controllers.

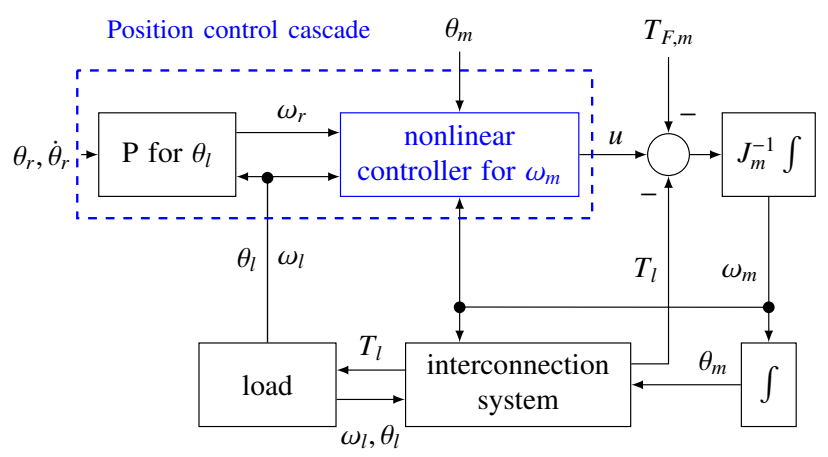

Figure 3: Position-velocity cascaded axis control. 


\subsection{Super-twisting sliding-mode control}

The first proposed axis positioning method is based on substituting the velocity PI controller with a STSMC, which is a second-order sliding-mode algorithm [62, 63]. Control schemes using sliding modes, first introduced in [64], have two very attractive features: disturbance rejection and finite-time convergence. The inherent robustness of sliding mode controllers against unknown dynamics and disturbances is achieved by using discontinuous terms in the control laws.

Over the past three decades there has been a growing interest in higher-order sliding mode algorithms. The motivation for studying higher-order SMCs is the effect of chattering on the actuators of the system induced by standard SMCs $[65,66,67,68]$. The application of the STSMC and its adaptive counterparts $[69,70]$ in motion control systems has been demonstrated in several works in the literature [71, 72] but high accuracy positioning has not been explicitly addressed.

For the regulation of the drive axis velocity error at zero the sliding variable $s$ is defined as

$$
s \triangleq e_{\omega}=\omega_{m}-\omega_{r}
$$

Its dynamics read:

$$
\begin{aligned}
\dot{s} & =\frac{1}{J_{m}}[\underbrace{u-T_{F, m}-\frac{K_{S}}{N}\left(\frac{\theta_{m}}{N}-\theta_{l}\right)-\frac{D_{S}}{N}\left(\frac{\omega_{m}}{N}-\omega_{l}\right)}_{\psi(x)}-J_{m} \dot{\omega}_{r}] \\
& =\frac{1}{J_{m}}\left[u+\psi(\boldsymbol{x})-J_{m} \dot{\omega}_{r}\right] .
\end{aligned}
$$

The STSMC control law is chosen as

$$
u=J_{m} \dot{\omega}_{r}+u_{S M C}
$$

where $u_{S M C}$ is given in [62]

$$
\begin{aligned}
u_{S M C} & =-k_{1}|s|^{\frac{1}{2}} \operatorname{sgn}(s)+v \\
\dot{v} & =-k_{2} \operatorname{sgn}(s)
\end{aligned}
$$

and the derivative of the velocity reference $\omega_{r}$ is calculated from (4.2).

Remark 1. The STSMC algorithm ensures that the system reaches the sliding manifold

$$
\mathcal{S}=\left\{\omega_{m}(t) \in \mathbb{R} \mid s\left(\omega_{m}(t)\right)=\dot{s}\left(\omega_{m}(t)\right)=0, \forall t \geq t_{r}\right\}
$$

in some finite-time $t_{r}$, provided that the control law gains are appropriately selected and that the first time-derivative of the matched perturbation $\psi(\boldsymbol{x})$ is bounded by a finite positive scalar $\xi$. However, this is not the case for the selected friction model since the $\operatorname{sgn}(\cdot)$ function is discontinuous at $\omega_{m}=0$. This means that when the motion changes direction, the system will leave the sliding surface and will reach it again very quickly for appropriate positive gains $k_{1}, k_{2}$.

Remark 2. The tuning of the STSMC gains, can be formulated as a Linear Matrix Inequality (LMI) problem [73] and a basic requirement is that the integral gain $k_{2}$ should be larger than the bound of the perturbation rate $\dot{\psi}(\boldsymbol{x})$. Specifically, selecting the gains as

$$
k_{2}>\xi, k_{1}=1.41 \sqrt{k_{2}+\xi}
$$

ensures finite-time convergence of $s$ to zero [74]. Even with a smooth approximation of the $\operatorname{sgn}(\cdot)$ function, such requirement leads to high values of $k_{1}, k_{2}$, making the algorithm more demanding in terms of control power and more sensitive to measurement noise.

\subsection{Nonlinear adaptive control}

Adaptive control has been an active field of research since the early sixties. Over this time, a large corpus of literature has been developed covering topics such as regulation and tracking of linear and nonlinear systems, disturbance rejection, etc. Tutorial presentations of the general adaptive control problem were given in $[75,76]$ on topics related to regulation, tracking, parameter estimation and robust control mostly for linear systems. The problem of adaptive control for a wider class of nonlinear systems was extensively studied in [77], presenting general design principles for NAC.

A geometric approach to the adaptive control and estimation problem was made in [78, 79] where the authors presented the main results of the I\&I-AC theory. The I\&I-AC designs were based on the task of finding a manifold on which the system has the required properties (stability, tracking, etc.) and then rendering it invariant by using appropriate control and adaptation laws. Application of these methods on electromechanical systems was demonstrated in [80] for robust velocity control of a Permanent Magnet Synchronous Motor (PMSM) and in [81] where trajectory tracking in a flexible joint manipulator with time-varying mechanical stiffness was pursued.

Two adaptive velocity controllers, namely the NAC and the I\&I-AC, are proposed for the motor velocity loop in the machine axis. The following assumption is made in both designs

\section{Assumption 4.1. (Constant unknown parameter vector)}

The unknown model parameters $\boldsymbol{\vartheta}$ are assumed to be constant or at least slowly varying, i.e. their time derivatives are considered to be zero

$$
\dot{\boldsymbol{\vartheta}}=\mathbf{0}
$$

\subsubsection{Velocity nonlinear adaptive controller}

The characteristic feature of this scheme is the adaptation of the coefficients in the friction model defined in (3.6), namely the motor Coulomb friction $T_{C, m}$, as well as the static and viscous friction coefficients $T_{S, m}$ and $\beta_{m}$, respectively. Due to the simplicity of the design, the shaft stiffness $K_{S}$ and damping coefficient $D_{S}$ can also be considered as uncertain parameters and be included in the adaptation algorithm. Let $\boldsymbol{\vartheta} \in \mathcal{P}$ be the unknown parameter vector defined as

$$
\boldsymbol{\vartheta} \triangleq\left[\begin{array}{lllll}
K_{S} & D_{S} & T_{C, m} & T_{S, m} & \beta_{m}
\end{array}\right]^{T}
$$


where the compact set $\mathcal{P}$ is defined as

$$
\mathcal{P}=\left[0, K_{S}^{\max }\right] \times\left[0, D_{S}^{\max }\right] \times\left[0, T_{C, m}^{\max }\right] \times\left[0, T_{S, m}^{\max }\right] \times\left[0, \beta_{m}^{\max }\right]
$$

and the superscript "max" denotes the maximum value the associated parameter can obtain. The dynamics of the velocity error are written as:

$$
\begin{aligned}
\dot{e}_{\omega} & =\frac{1}{J_{m}}\left[u-\frac{K_{S}}{N}\left(\frac{1}{N} \theta_{m}-\theta_{l}\right)-\frac{D_{S}}{N}\left(\frac{1}{N} \omega_{m}-\omega_{l}\right)-T_{F, m}\right]-\dot{\omega}_{r} \\
& =\frac{1}{J_{m}}\left[u+\boldsymbol{\phi}^{T}(\boldsymbol{x}) \boldsymbol{\vartheta}\right]-\dot{\omega}_{r}
\end{aligned}
$$

where $\boldsymbol{x}=\left[\begin{array}{llll}\omega_{m} & \theta_{m} & \omega_{l} & \theta_{l}\end{array}\right]^{T}$ is the drive train state vector, and $\boldsymbol{\phi}(\boldsymbol{x})$ is the regressor function defined as

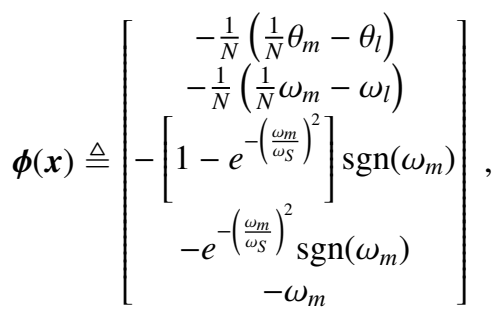

with $\omega_{S}$ being the identified Stribeck velocity for the drive motor.

Proposition 1. The control law

$$
u=-\boldsymbol{\phi}^{T}(\boldsymbol{x}) \hat{\boldsymbol{\vartheta}}-k e_{\omega}+J_{m} \dot{\omega}_{r}
$$

together with the adaptation laws

$$
\dot{\hat{\boldsymbol{\vartheta}}}=\boldsymbol{\Gamma} \boldsymbol{\phi}(\boldsymbol{x}) e_{\omega}
$$

where $k$ is a positive real number and $\boldsymbol{\Gamma}$ a $5 \times 5$ positive definite real matrix, ensure that the velocity tracking error $e_{\omega}$ with dynamics given in Equation (4.10) converges to the origin $e_{\omega}^{*}=0$ as $t \rightarrow \infty$, i.e.

$$
\lim _{t \rightarrow \infty} e_{\omega}(t)=0 .
$$

Moreover, the parameter estimation error $\tilde{\boldsymbol{\vartheta}} \triangleq \boldsymbol{\vartheta}-\hat{\boldsymbol{\vartheta}}$ remains bounded for all future times.

Proof. Denoting an estimate of the real parameter vector with $\hat{\boldsymbol{\vartheta}}$, the equivalence-principle control law [77]

$$
u=-\boldsymbol{\phi}^{T}(\boldsymbol{x}) \hat{\boldsymbol{\vartheta}}+J_{m} \dot{\omega}_{r}-k e_{\omega}
$$

will give the following tracking error dynamics

$$
\dot{e}_{\omega}=\frac{1}{J_{m}}\left[\boldsymbol{\phi}^{T}(\boldsymbol{x}) \tilde{\boldsymbol{\vartheta}}-k e_{\omega}\right] .
$$

Consider the following positive definite Lyapunov function candidate

$$
V=\frac{1}{2} J_{m} e_{\omega}^{2}+\frac{1}{2} \tilde{\boldsymbol{\vartheta}}^{T} \boldsymbol{\Gamma}^{-1} \tilde{\boldsymbol{\vartheta}}
$$

Noting that $\dot{\boldsymbol{\vartheta}}=\mathbf{0}$, the time derivative of $V$ along the trajectories of (4.15) reads:

$$
\dot{V}=-k e_{\omega}^{2}+e_{\omega} \boldsymbol{\phi}^{T}(\boldsymbol{x}) \tilde{\boldsymbol{\vartheta}}-\tilde{\boldsymbol{\vartheta}}^{T} \boldsymbol{\Gamma}^{-1} \dot{\hat{\boldsymbol{\vartheta}}} .
$$

Then substituting (4.13) in (4.16) yields

$$
\dot{V}=-k e_{\omega}^{2} \leq 0
$$

which implies that $V$ is non-increasing. Since $V\left(e_{\omega}(t), \tilde{\boldsymbol{\vartheta}}(t)\right) \geq$ $0, \forall t \geq 0$, it follows that $V$ is bounded and, by extension, $e_{\omega}(t), \tilde{\boldsymbol{\vartheta}}(t)$ are also bounded for all future times. Taking the second time-derivative of $V$ leads to

$$
\ddot{V}=-\frac{2 k}{J_{m}}\left[e_{\omega} \boldsymbol{\phi}^{T}(\boldsymbol{x}) \tilde{\boldsymbol{\vartheta}}-k e_{\omega}^{2}\right]
$$

Boundedness of $e_{\omega}, \tilde{\boldsymbol{\vartheta}}$ implies that $\ddot{V}$ is also bounded and, as a result, that $\dot{V}$ is uniformly continuous. Then, since $\lim _{t \rightarrow \infty} V(t)=$ $V(\infty) \leq V(0)$ is finite, by applying Barbălat's lemma [77, Lemma A.6] it is shown that $\lim _{t \rightarrow \infty} \dot{V}\left(e_{\omega}(t)\right)=0$. From (4.17) it can be seen that

$$
\lim _{t \rightarrow \infty} e_{\omega}(t)=0,
$$

which completes the proof.

It should be noted that parameter convergence to the real values is not guaranteed, unless $\boldsymbol{\phi}(\boldsymbol{x})$ is persistently exciting [76], i.e. unless there exist constants $\alpha_{0}, \alpha_{1}, T_{0}>0$ such that

$$
\alpha_{0} \boldsymbol{I} \leq \frac{1}{T_{0}} \int_{t}^{t+T_{0}} \boldsymbol{\phi}(\tau) \boldsymbol{\phi}^{T}(\tau) d \tau \leq \alpha_{1} \boldsymbol{I}
$$

\subsubsection{Velocity Immersion and Invariance adaptive controller}

The dynamics for velocity tracking error $e_{\omega}$ are given by

$$
\dot{e}_{\omega}=\frac{1}{J_{m}}\left[u+\boldsymbol{\phi}^{T}(\boldsymbol{x}) \boldsymbol{\vartheta}-\frac{1}{N} T_{l}\right]-\dot{\omega}_{r}
$$

where now the unknown parameter vector is defined as

$$
\boldsymbol{\vartheta} \triangleq\left[\begin{array}{llll}
T_{C, m} & T_{S, m} & \beta_{m} & b
\end{array}\right]^{T}
$$

with

$$
\boldsymbol{\vartheta} \in \boldsymbol{Q}=\left[0, T_{C, m}^{\max }\right] \times\left[0, T_{S, m}^{\max }\right] \times\left[0, \beta_{m}^{\max }\right] \times\left[-b^{\max }, b^{\max }\right] .
$$

The regressor function $\boldsymbol{\phi}(\boldsymbol{x})$ is defined as

$$
\boldsymbol{\phi}(\boldsymbol{x}) \equiv \boldsymbol{\phi}\left(\omega_{m}\right) \triangleq\left[\begin{array}{c}
-\left[1-e^{-\left(\frac{\omega_{m}}{\omega_{S}}\right)^{2}}\right] \operatorname{sgn}\left(\omega_{m}\right) \\
-e^{-\left(\frac{\omega_{m}}{\omega_{S}}\right)^{2}} \operatorname{sgn}\left(\omega_{m}\right) \\
-\omega_{m} \\
-1
\end{array}\right]
$$

and $T_{l}$ is defined in Equation (3.5). Since only the varying friction is considered unknown, the shaft parameters are not included in the design. This considerably simplifies the complexity of the control algorithm. Parameter $b$ serves as an additional integral term that can compensate for minor variations of the interconnection torque $T_{l}$ due to small uncertainties in the shaft parameters. 
If $\hat{\boldsymbol{\vartheta}}$ is an estimate of the real parameter vector, the approach in the I\&I-AC is to design a control input and an update law for $\hat{\boldsymbol{\vartheta}}$, which makes the manifold

$$
\mathcal{M}=\left\{(\boldsymbol{x}, \hat{\boldsymbol{\vartheta}}) \in \mathbb{R}^{4} \times Q \mid \hat{\boldsymbol{\vartheta}}-\boldsymbol{\vartheta}+\boldsymbol{h}(\boldsymbol{x})=\mathbf{0}\right\}
$$

invariant, where $\boldsymbol{h}(\boldsymbol{x})$ is a known function to be defined. Once the system is on this manifold, the unknown parameter vector $\vartheta$ can be calculated by $\boldsymbol{\vartheta}=\hat{\boldsymbol{\vartheta}}+\boldsymbol{h}(\boldsymbol{x})$. Note that the design of $\boldsymbol{h}(\boldsymbol{x})$ is also included in this process.

Theorem 4.1. The control law

$$
u=\frac{1}{N} T_{l}-\boldsymbol{\phi}^{T}\left(\omega_{m}\right)\left(\hat{\boldsymbol{\vartheta}}+\boldsymbol{h}\left(\omega_{m}\right)\right)-k_{I I} e_{\omega}+J_{m} \dot{\omega}_{r}
$$

together with the adaptation laws

$$
\dot{\hat{\boldsymbol{\vartheta}}}=\frac{\partial \boldsymbol{h}}{\partial \omega_{m}} \frac{1}{J_{m}}\left(k_{I I} e_{\omega}-J_{m} \dot{\omega}_{r}\right)
$$

with $k_{I I}$ being a positive real number and the real function $\boldsymbol{h}$ : $\mathbb{R} \rightarrow \mathbb{R}^{4}$ being defined as

$$
\boldsymbol{h}\left(\omega_{m}\right)=J_{m} \boldsymbol{\Gamma}_{\boldsymbol{I I}}\left[\begin{array}{c}
-\left|\omega_{m}\right|+\frac{\omega_{S} \sqrt{\pi}}{2} \operatorname{erf}\left(\frac{\omega_{m}}{\omega_{S}}\right) \operatorname{sgn}\left(\omega_{m}\right) \\
-\frac{\omega_{S} \sqrt{\pi}}{2} \operatorname{erf}\left(\frac{\omega_{m}}{\omega_{S}}\right) \operatorname{sgn}\left(\omega_{m}\right) \\
-\frac{1}{2} \omega_{m}^{2} \\
-\omega_{m}
\end{array}\right]
$$

where $\boldsymbol{\Gamma}_{I I}$ a $4 \times 4$ is a positive definite real matrix and $\operatorname{erf}(\cdot)$ is the error function, ensure that the velocity tracking error $e_{\omega}$ with dynamics given in Equation (4.19), as well as the parameter estimation error $\tilde{\boldsymbol{\vartheta}}$ remain bounded for all future times. Additionally, if $\phi\left(\omega_{m}\right)$ is persistently exciting, then

$$
\lim _{t \rightarrow \infty}\left[\begin{array}{c}
e_{\omega}(t) \\
\tilde{\boldsymbol{\vartheta}}(t)
\end{array}\right]=\mathbf{0}
$$

Proof. Similarly to the case of NAC, applying the equivalence principle control law yields the following velocity error dynamics

$$
\dot{e}_{\omega}=\frac{1}{J_{m}}\left[-k_{I I} e_{\omega}-\boldsymbol{\phi}^{T}\left(\omega_{m}\right)(\hat{\boldsymbol{\vartheta}}+\boldsymbol{h}(\boldsymbol{x})-\boldsymbol{\vartheta})\right] .
$$

The new feature when compared to NAC is the "parameter offset" function $\boldsymbol{h}(\boldsymbol{x})$, which quantifies the deviation of the estimated parameter vector $\hat{\boldsymbol{\vartheta}}$ from its real value. The objective is to find a function $\boldsymbol{h}(\boldsymbol{x}) \equiv \boldsymbol{h}\left(\omega_{m}\right)$ and an update law $\dot{\hat{\boldsymbol{\vartheta}}}=\boldsymbol{w}(\boldsymbol{x}, \hat{\boldsymbol{\vartheta}})$ such that the manifold $\mathcal{M}$ is attractive invariant. To achieve this, the off-the-manifold coordinate

$$
\boldsymbol{z} \triangleq \hat{\boldsymbol{\vartheta}}-\boldsymbol{\vartheta}+\boldsymbol{h}\left(\omega_{m}\right)
$$

is defined. Its dynamics read

$$
\begin{aligned}
\dot{\boldsymbol{z}} & =\dot{\hat{\boldsymbol{\vartheta}}}+\frac{\partial \boldsymbol{h}}{\partial \omega_{m}} \dot{\omega}_{m}=\boldsymbol{w}(\boldsymbol{x}, \hat{\boldsymbol{\vartheta}})+\frac{\partial \boldsymbol{h}}{\partial \omega_{m}} \frac{1}{J_{m}}\left[u+\boldsymbol{\phi}^{T}\left(\omega_{m}\right) \boldsymbol{\vartheta}-\frac{1}{N} T_{l}\right] \\
& =\boldsymbol{w}(\boldsymbol{x}, \hat{\boldsymbol{\vartheta}})+\frac{\partial \boldsymbol{h}}{\partial \omega_{m}} \frac{1}{J_{m}}\left[-\boldsymbol{\phi}^{T}\left(\omega_{m}\right) z-k_{I I} e_{\omega}+J_{m} \dot{\omega}_{r}\right]
\end{aligned}
$$

where Assumption (4.1) and Equations (4.23), (4.27) were used. Selecting

$$
\boldsymbol{w}(\boldsymbol{x}, \hat{\boldsymbol{\vartheta}})=\frac{\partial \boldsymbol{h}}{\partial \omega_{m}} \frac{1}{J_{m}}\left(k_{I I} e_{\omega}-J_{m} \dot{\omega}_{r}\right)
$$

the dynamics of the $z$-coordinate become

$$
\dot{z}=-\frac{1}{J_{m}} \frac{\partial \boldsymbol{h}}{\partial \omega_{m}} \boldsymbol{\phi}^{T}\left(\omega_{m}\right) z
$$

Selecting $\boldsymbol{h}$ such that

$$
\frac{\partial \boldsymbol{h}}{\partial \omega_{m}}=J_{m} \boldsymbol{\Gamma}_{\boldsymbol{I I}} \boldsymbol{\phi}\left(\omega_{m}\right)
$$

suggests

$$
\boldsymbol{h}\left(\omega_{m}\right)=J_{m} \boldsymbol{\Gamma}_{\boldsymbol{I I}}\left[\begin{array}{c}
-\left|\omega_{m}\right|+\frac{\omega_{S} \sqrt{\pi}}{2} \operatorname{erf}\left(\frac{\omega_{m}}{\omega_{S}}\right) \operatorname{sgn}\left(\omega_{m}\right) \\
-\frac{\omega_{S} \sqrt{\pi}}{2} \operatorname{erf}\left(\frac{\omega_{m}}{\omega_{S}}\right) \operatorname{sgn}\left(\omega_{m}\right) \\
-\frac{1}{2} \omega_{m}^{2} \\
-\omega_{m}
\end{array}\right] .
$$

Substituting $\boldsymbol{h}$ in (4.30) yields

$$
\dot{z}=-\boldsymbol{\Gamma}_{\boldsymbol{I I}} \boldsymbol{\phi}\left(\omega_{m}\right) \boldsymbol{\phi}^{T}\left(\omega_{m}\right) z
$$

which verifies that the solutions $z(t)$ of (4.33) are bounded since $\boldsymbol{\phi}\left(\omega_{m}\right) \boldsymbol{\phi}^{T}\left(\omega_{m}\right)$ is positive semidefinite. To prove that $\mathcal{M}$ is attractive and invariant, it is sufficient to show that

$$
\lim _{t \rightarrow \infty} z(t)=\lim _{t \rightarrow \infty} \dot{z}(t)=0 .
$$

Consider the real-valued positive-definite Lyapunov function candidate $V: \mathbb{R}^{4} \rightarrow \mathbb{R}$ defined as:

$$
V(z(t))=\frac{1}{2} z(t)^{T} z(t) \geq 0
$$

for which it holds

$$
\frac{1}{4} z(t)^{T} z(t) \leq V(z(t)) \leq z(t)^{T} z(t), \forall t \geq 0 .
$$

Its time derivative along the trajectories of (4.33) is given by

$$
\dot{V}(z(t))=-z^{T}(t) \boldsymbol{\phi}\left(\omega_{m}\right) \boldsymbol{\phi}^{T}\left(\omega_{m}\right) z(t) \leq 0
$$

which implies that $V(z(t))$ is not increasing for all $t \geq 0$. Consequently, boundedness of $V$ implies boundedness of $z(t)$ and, by extension, of $\omega_{m}(t)$, i.e. $z(t), \omega_{m}(t) \in \mathcal{L}_{\infty}$. Moreover, it can also be concluded from (4.33) that $\dot{z}(t) \in \mathcal{L}_{\infty}$. Integrating (4.35) from both sides yields

$$
0 \leq \int_{0}^{\infty} z^{T}(t) \phi\left(\omega_{m}(t)\right) \boldsymbol{\phi}^{T}\left(\omega_{m}(t)\right) z(t) d t \leq V(z(0))-V(z(\infty)) .
$$

This means that if $\phi\left(\omega_{m}(t)\right)$ is persistently exciting (see (4.18)), then $z(t) \in \mathcal{L}_{2}, \forall t \geq 0$. Then applying Barbălat's lemma [77, Lemma A6] gives

$$
\lim _{t \rightarrow \infty} z(t)=\mathbf{0}
$$


From (4.30) it also follows that $\lim _{t \rightarrow \infty} \dot{z}(t)=\mathbf{0}$, which proves that $\mathcal{M}$ is invariant. Moreover, the closed-loop velocity error dynamics

$$
\dot{e}_{\omega}=-\frac{1}{J_{m}}\left[k_{I I} e_{\omega}+\boldsymbol{\phi}\left(\omega_{m}\right) \boldsymbol{\phi}^{T}\left(\omega_{m}\right) z\right]
$$

can be seen as the cascaded interconnection of a convergent system ( $z$-dynamics) with a Uniformly Globally Exponentially Stable (UGES) system (unperturbed velocity error dynamics):

$$
\begin{aligned}
\dot{z} & =-\frac{1}{J_{m}} \boldsymbol{\phi}\left(\omega_{m}\right) \boldsymbol{\phi}^{T}\left(\omega_{m}\right) z \\
\dot{e}_{\omega} & =-\frac{1}{J_{m}} k_{I I} e_{\omega} .
\end{aligned}
$$

Since $e_{\omega}$ is Input-to-State Stable (ISS) with respect to $z$ (see Appendix A for a proof), boundedness of $z(t)$ implies boundedness of the velocity tracking error, while convergence of $z(t)$ to zero implies that $\lim _{t \rightarrow \infty} e_{\omega}(t)=0$. Lastly, since $z(t), \boldsymbol{h}\left(\omega_{m}\right)$ are bounded, it follows from (4.27) that the parameter estimation error $\tilde{\boldsymbol{\vartheta}}$ is also bounded and it converges to zero, when $z(t)$ vanishes, i.e. if the regressor function is persistently exciting. This completes the proof.

Remark 3. It should be noted that Equations (4.5), (4.10) and (4.19) refer to the same velocity error dynamics. Their differences relate to the specific parametrization used in each design.

Remark 4. Including the adaptation of the shaft parameters $K_{S}, D_{S}$ would have made the design of I\&I-AC, far more complicated. This is due to the fact that the regressor function $\phi$ in (4.21) - and by extension - function $\boldsymbol{h}$, would depend on the entire state vector $\boldsymbol{x}$ instead of just the motor angular velocity $\omega_{m}$. This means that instead of the Partial Differential Equation (PDE) in (4.31), we would have to solve a $5 \times 5$ system of PDEs, which is not a trivial task and it may not be possible to obtain a closed-form analytical solution.

Remark 5. In both designs, the existence of the closed-loop system solutions requires $\boldsymbol{\phi}(\boldsymbol{x})$ to be locally Lipschitz. This does not hold since its derivative is not bounded at 0 . Since, however, the regressor function is continuously differentiable everywhere except for a finite number of points corresponding to $\omega_{m}=0$, the solutions of closed-loop system can be understood in the sense of Filippov [82, 83].

Remark 6. For enhancing numerical robustness against noise, the function $\operatorname{sgn}(\cdot)$ is approximated with a sigmoid function, e.g. $\operatorname{sgn}(y) \approx 2 / \pi \cdot \arctan (\alpha y) \triangleq \rho(\alpha, y)$, where $\alpha$ is a positive number denoting the slope of the function near 0 . The motivation behind this approximation is the fact that in the case of Coulomb friction, the sensitivity of the $\operatorname{sgn}(\cdot)$ function to noise-inflicted measurements can induce friction-compensating torques in the wrong direction and with high frequency, leading to actuator damage or even instability. For large values of the scaling factor $\alpha$ (e.g. $\alpha \geq 100), \rho(\alpha, y)$ approximates $\operatorname{sgn}(y)$ with sufficient accuracy for the applications and $\lim _{\alpha \rightarrow \infty} \rho(\alpha, y)=\operatorname{sgn}(y)$. Similarly, the error function defined as $\operatorname{erf}(y)=2 / \sqrt{\pi} \cdot \int_{0}^{y} e^{-\tau^{2}} d \tau$ can be approximated by $\operatorname{erf}(y) \approx$ $\tanh (y)=\left(e^{y}-e^{-y}\right) /\left(e^{y}+e^{-y}\right)$.

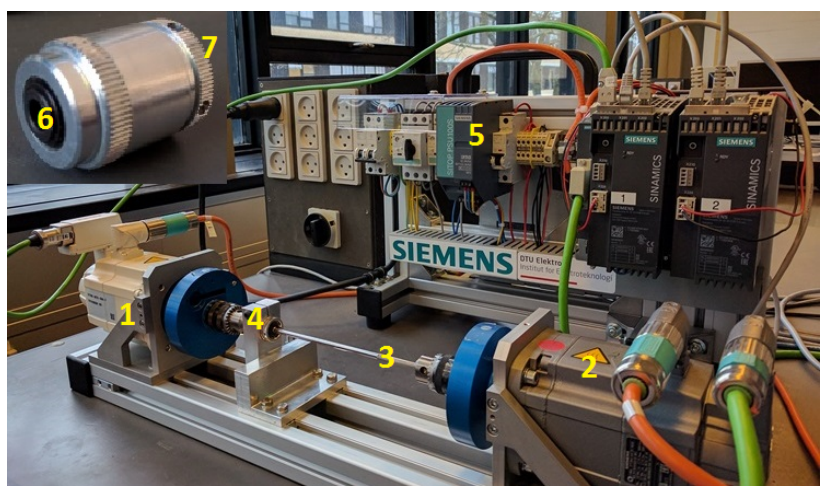

Figure 4: Experimental setup: (1) 1FT7 drive PMSM, (2) 1FT7 load PMSM, (3) shaft, (4) friction component base, (5) Siemens SINAMICS S120 converter, (6) shaft housing, (7) friction adjustment ring.

\section{Experiments and evaluation}

\subsection{Experimental setup}

The experimental setup used in this study consisted of two Siemens 1FT7042-5AF70 PMSMs (one acting as pure inertia) connected through a steel shaft which included a Vari-tork 279.25.22 adjustable-friction clutch [84]. This clutch allows increasing the Coulomb friction on the motor side through an adjustable ring. Both motors were equipped with 22-bit incremental position encoders, which corresponds to approximately $1.5 \mu \mathrm{rad}$ measurement accuracy. The motors velocities were provided by the commissioning software STARTER via numerical differentiation of the position measurements, and were inflicted with zero mean white Gaussian noise with variance $\sigma_{\text {meas }}^{2} \approx 8.1 \cdot 10^{-5} \mathrm{rad}^{2} / \mathrm{s}^{2}$. The drive converter was integrated in a SINAMICS S120 control unit, which handled the current control loops. All the algorithms were implemented as custom software updates in STARTER at sampling time $125 \mu \mathrm{s}$. The entire drive train with the friction component are shown in Figure 4.

\subsection{Test scenarios}

Fifteen test scenarios for evaluating the performance of the control algorithms were considered in this study. The tests included sinusoidal motion profiles $\theta_{r}(t)=\Theta_{0} \sin \left(2 \pi f_{r} t\right)$ at three frequencies $f_{r}$ and under five different Coulomb fiction cases. The increase in the Coulomb friction ranged from $215 \%$ to $900 \%$ of its nominal value, with the extreme cases $(615 \%$ and $900 \%$ increase) being considered for testing the performance limits of the controllers.

The motivation for choosing sinusoidal position reference relates to the fact that the most significant positioning errors in machine tools occur during axis motion reversal, where the sudden change in Coulomb friction causes spikes in the position error profile [1]. An additional reason behind this choice pertains to evaluating the positioning accuracy when a machine performs a circular contouring task, which is a standard test in machine tools [85]. Finally, since the focus of this study is on the friction-resilience of the proposed controllers, the reference frequencies were in the range $0.1-2 \mathrm{~Hz}$ to reflect very low speeds, where the nonlinear friction phenomena dominate, as 
Table 2: Test scenarios. The notation $F_{i}$ with $i=1, \ldots, 4$ denote the four different friction cases.

\begin{tabular}{ccccc} 
No & $\Theta_{0}(\mathrm{rad})$ & $T_{C, m}(\mathrm{~N} \mathrm{~m})$ & $f_{r}(\mathrm{~Hz})$ & Increase in friction \\
\hline 1 & 1 & 0.035 & 0.1 & 0 (nominal) \\
2 & 1 & 0.035 & 0.5 & 0 (nominal) \\
3 & 1 & 0.035 & 2 & 0 (nominal) \\
\hline 4 & 1 & 0.11 & 0.1 & $215 \%\left(F_{1}\right)$ \\
5 & 1 & 0.11 & 0.5 & $215 \%\left(F_{1}\right)$ \\
6 & 1 & 0.11 & 2 & $215 \%\left(F_{1}\right)$ \\
\hline 7 & 1 & 0.15 & 0.1 & $330 \%\left(F_{2}\right)$ \\
8 & 1 & 0.15 & 0.5 & $330 \%\left(F_{2}\right)$ \\
9 & 1 & 0.15 & 2 & $330 \%\left(F_{2}\right)$ \\
\hline 10 & 1 & 0.25 & 0.1 & $615 \%\left(F_{3}\right)$ \\
11 & 1 & 0.25 & 0.5 & $615 \%\left(F_{3}\right)$ \\
12 & 1 & 0.25 & 2 & $615 \%\left(F_{3}\right)$ \\
\hline 13 & 1 & 0.35 & 0.1 & $900 \%\left(F_{4}\right)$ \\
14 & 1 & 0.35 & 0.5 & $900 \%\left(F_{4}\right)$ \\
15 & 1 & 0.35 & 2 & $900 \%\left(F_{4}\right)$ \\
\hline
\end{tabular}

well as frequent motion reversals. The test scenarios are summarised in Table 2.

\subsection{Controllers comparison criteria}

Table 3: Controllers comparison criteria.

\begin{tabular}{lcl} 
Criterion & Definition/description & Focus \\
\hline MAE & $\sup _{t_{0} \leq t \leq t_{0}+T}\left|e_{\theta}(t)\right|$ & Error peak \\
\hline CP & $\frac{1}{T} \int_{t_{0}+T}^{t_{0}+T} u^{2}(t) d t$ & Control usage \\
\hline ECP & MAE.CP & Efficiency \\
\hline MAPE & $\sup _{t_{0} \leq t \leq t_{0}+T}\left|e_{\phi}(t)\right|$ with & Phase lag \\
& $e_{\phi}(t)=\phi_{r e f}(t)-\phi_{\text {real }}(t)$ & \\
& $\phi_{\text {ref }}(t)=\tan ^{-1}\left(\frac{\theta_{y}^{r}(t)}{\theta_{x}^{r}(t)}\right)$ & \\
& $\phi_{\text {real }}(t)=\tan ^{-1}\left(\frac{\theta_{y}(t)}{\theta_{x}(t)}\right)$ & \\
& $\theta_{x, y}(t)=\theta_{l}(t) / \Theta_{0}$ & \\
& $\theta_{x}^{r}(t)=\sin \left(2 \pi f_{r} t\right)$ & \\
& $\theta_{y}^{r}(t)=\cos \left(2 \pi f_{r} t\right)$ & \\
\hline CI & $N_{p}+N_{s}$ & Design complexity \\
\hline
\end{tabular}

The quantitative criteria for evaluating the controllers' performance, introduced in [42], namely the Maximum Absolute Error (MAE), Control Power (CP), Error-Control Power product (ECP), Maximum Absolute Phase Error (MAPE) and Complexity Index (CI) are summarised in Table 3 , where $N_{p}, N_{s}$ denote the number of tunable parameters and required signals for each design, respectively. Evaluation of MAPE is very important especially in machines with more than two axes because it is indicative of delays in positioning that may result in a distorted contour [1]. Since the test rig consists of only one axis, the experiments were repeated with the position reference being shifted by $\pi / 2$, so as to emulate a 2 -axes trajectory $\theta_{x}-\theta_{y}$.

\subsection{Controller tuning}

The controllers' parameters were selected according to Table 4. To ensure a fair comparison, the controllers were tuned in the nominal case (with the friction clutch dismounted from the test rig), such that the maximum absolute positioning error is below $5 \cdot 10^{-3} \mathrm{rad}$ at $0.5 \mathrm{~Hz}$ for all control schemes. This follows the idea that in real applications the control loops are tuned according to the state of the physical system at the time of the commissioning since future wear cannot be quantified. The PI gains were selected after experimental tests by gradually increasing the proportional gain $k_{p}$ and then decreasing the reset time $T_{n}$. The criterion was to reduce MAE as much as possible for the nominal case at $0.5 \mathrm{~Hz}$, without compromising the stability of the closed-loop system $\left(k_{p} \geq 1.8\right.$ with MAE at best $\left.3.8 \cdot 10^{-3} \mathrm{rad}\right)$.

Table 4: Controller parameters values.

\begin{tabular}{clc} 
Symbol & Description & Value \\
\hline \multicolumn{2}{c}{ Outer loop P } & \\
\hline \multicolumn{2}{c}{$k_{p o s}$} & Proportional gain \\
\hline PI & & \\
\hline$k_{p}$ & Proportional gain & 0.9 \\
$T_{n}$ & Integral reset time & 0.06 \\
\hline STSMC & & \\
\hline$k_{1}$ & Switching gain & 0.9 \\
$k_{2}$ & Integral sw. gain & 75 \\
\hline NAC & & \\
\hline$k$ & Proportional gain & 0.6 \\
$\operatorname{diag}(\mathbf{\Gamma})$ & Adaptation gains & {$\left[10^{-5}, 0.5,0.12,0.12,4.5\right]$} \\
$\hat{\boldsymbol{\vartheta}}_{\mathbf{0}}$ & Initial conditions & {$[17,0.003,0,0,0.0008]^{T}$} \\
\hline $\mathbf{I} \mathbf{I}-\mathbf{A C}$ & & \\
\hline$k_{I I}$ & Proportional gain & 0.6 \\
$\operatorname{diag}\left(\boldsymbol{\Gamma}_{\boldsymbol{I I}}\right)$ & Adaptation gains & {$[0.1203,0.012,0.012,0.012]$} \\
$\hat{\boldsymbol{\vartheta}}_{\mathbf{0}}$ & Initial conditions & {$[0.003,0,0,0.0008]^{T}$} \\
\hline & &
\end{tabular}

The outer loop position P controller gain was kept the same in all schemes, and the proportional gains of all three nonlinear velocity controllers $\left(k_{1}, k, k_{I I}\right)$ were chosen to be smaller than or equal to the PI proportional gain $k_{p}$. The adaptation gains in NAC and I\&I-AC were iteratively selected by starting from small values and increasing until parameter estimation could be achieved as fast as possible without significant overshoots in the estimation signals. Since, friction model mismatches affect the tracking error $e_{\omega}$, they also show up in the estimation signals. 


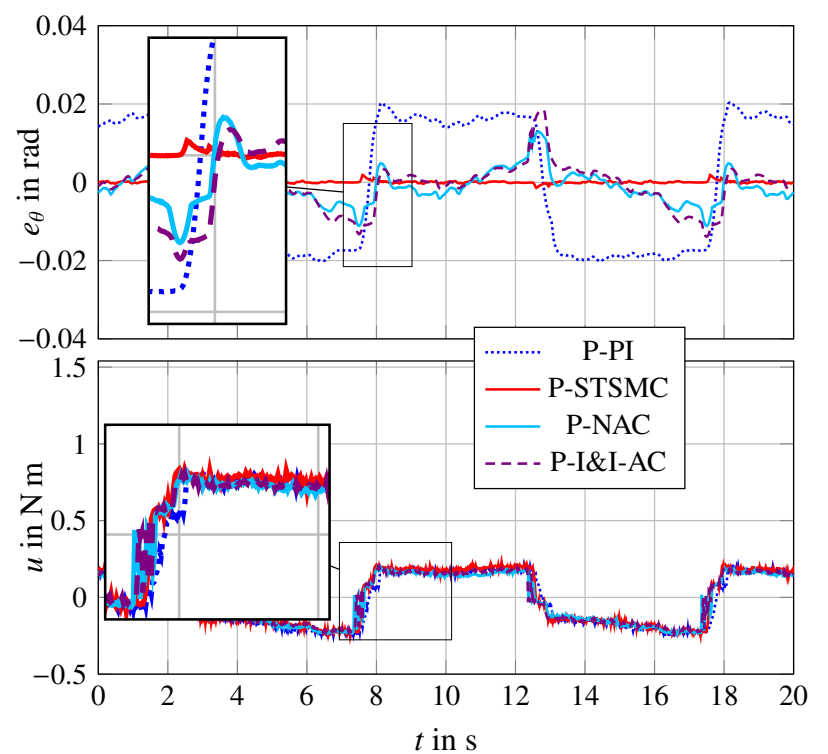

Figure 5: Positioning errors and torque commands for Test 7. No excessive STSMC chatter is present in the command torque.

This can be immediately seen by examining the adaptation laws in Equations (4.13) and (4.29). The larger the adaptation gains, the more significant the effect of the model mismatches on the estimation signals. Therefore, the selection of the final values was made by considering the trade-off between parameter convergence speed, as well as, overshoot size and amount of chatter in the estimation signals. Finally, the selection of the initial parameter estimates $\hat{\boldsymbol{\vartheta}}_{\mathbf{0}}$ is also important, since a high initial parameter error gives a substantially large initial velocity error, which may cause the parameter estimates to overshoot and reach values associated to unwanted system behaviours. This can be alleviated to some extent by using parameter projection algorithms [77] at the cost, however, of risking that already converged parameters move away from their correct value and settle to another one.

\subsection{Results}

The controllers were tested for 7 minutes in each experiment and their performance was evaluated during the last $20 \mathrm{~s}$. This was done to allow the parameters in the adaptation schemes to settle and also to avoid including initial errors in the analysis due to the setpoint ramping up.

Table 5 shows the MAE for all the different controllers during all tests. Already from the first friction case (Test 4), the P-PI scheme degrades in performance with MAE approximately four times higher than the prescribed accuracy limit (10 mrad). The P-STSMC performs better than all the controllers with the lowest MAE, and also below the accuracy limit. In the case of the slowest motion profile, the P-STSMC achieves a MAE almost 10 times lower than the specified performance. The adaptive controllers outperform the conventional P-PI cascade in all cases, except at low frequencies and with extreme friction (Tests 10 and 13). This is due to the fact that the friction adjustment ring inherently has a dead zone of approximately $0.035 \mathrm{rad}$ between the shaft housing and outer cylinder. The induced

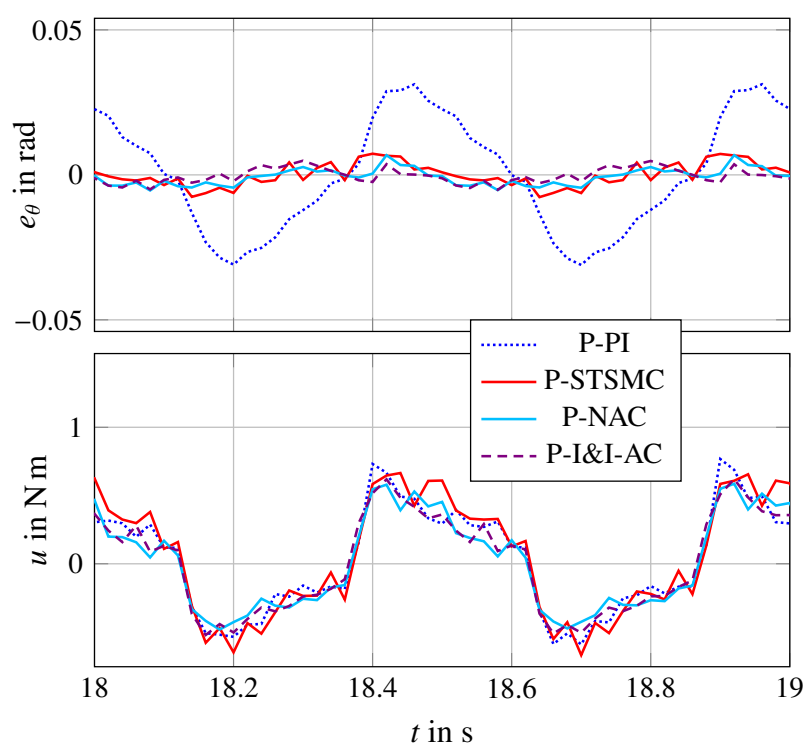

Figure 6: Positioning errors and torque commands for Test 9. Faster frequency facilitates better performance for NAC/I\&I due to richer excitation.

backlash causes a step change in the friction parameters (from nominal inside the dead zone to the increased value outside of the dead zone) that is too fast the adaptation schemes, leading to increased peak errors during motion reversal.

Table 5: MAE in mrad for all controllers in all scenarios. The notation $F_{i}$ with $i=1, \ldots, 4$ denote the four different friction cases.

\begin{tabular}{cccccc}
\hline Controller & nominal & $F_{1}$ & $F_{2}$ & $F_{3}$ & $F_{4}$ \\
\hline MAE in mrad at $0.1 \mathrm{~Hz}$ \\
STSMC & 0.9 & 1.5 & 1.9 & 2.9 & 3.8 \\
NAC & 7.8 & 8.3 & 12.9 & 20.2 & 40.7 \\
I\&I-AC & 7.8 & 12.5 & 18.5 & 33.3 & 43 \\
\hline MAE in mrad at & $0.5 \mathrm{~Hz}$ & & \\
\hline PI & 4.9 & 15.6 & 17 & 23.7 & 37.2 \\
STSMC & 2 & 3 & 4.1 & 7.5 & 10.9 \\
NAC & 2.2 & 7.5 & 12.2 & 21.1 & 35 \\
I\&I-AC & 4.9 & 7.2 & 13.2 & 20.3 & 34.3 \\
\hline MAE in mrad at & $2 \mathrm{~Hz}$ & & \\
\hline PI & 12.3 & 20.1 & 21.8 & 31.6 & 45.7 \\
STSMC & 7 & 6.8 & 7.2 & 10.6 & 12.3 \\
NAC & 8.7 & 6.8 & 6.9 & 7.1 & 7.3 \\
\hline I I-AC & 5.8 & 6.7 & 6 & 5.7 & 9.2 \\
\hline
\end{tabular}

This effect of the backlash is minimized at faster references, where the controllers demonstrate higher accuracy as shown by comparing Figures 5 and 6. Looking at the same figures, one can see that the control signals of all the controllers were approximately at the same levels, which implies that the efficiency of the P-PI was lower than that of the nonlinear solutions.

A visualization of the MAE and MAPE of the controllers can 
$0.1 \mathrm{~Hz}$
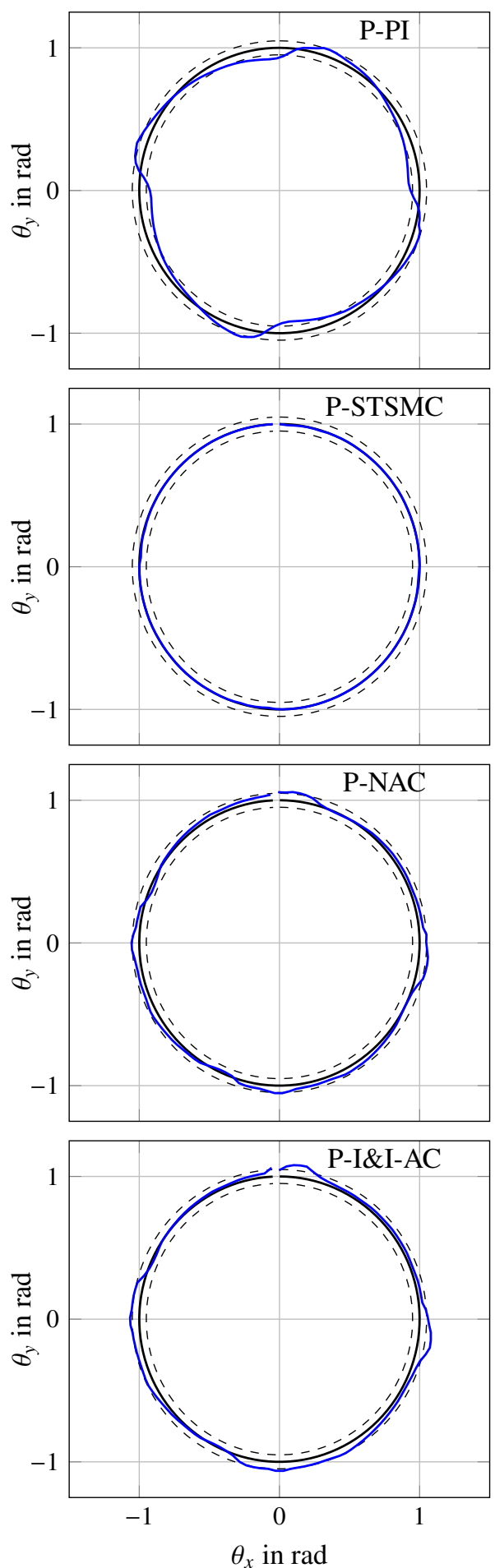

$0.5 \mathrm{~Hz}$
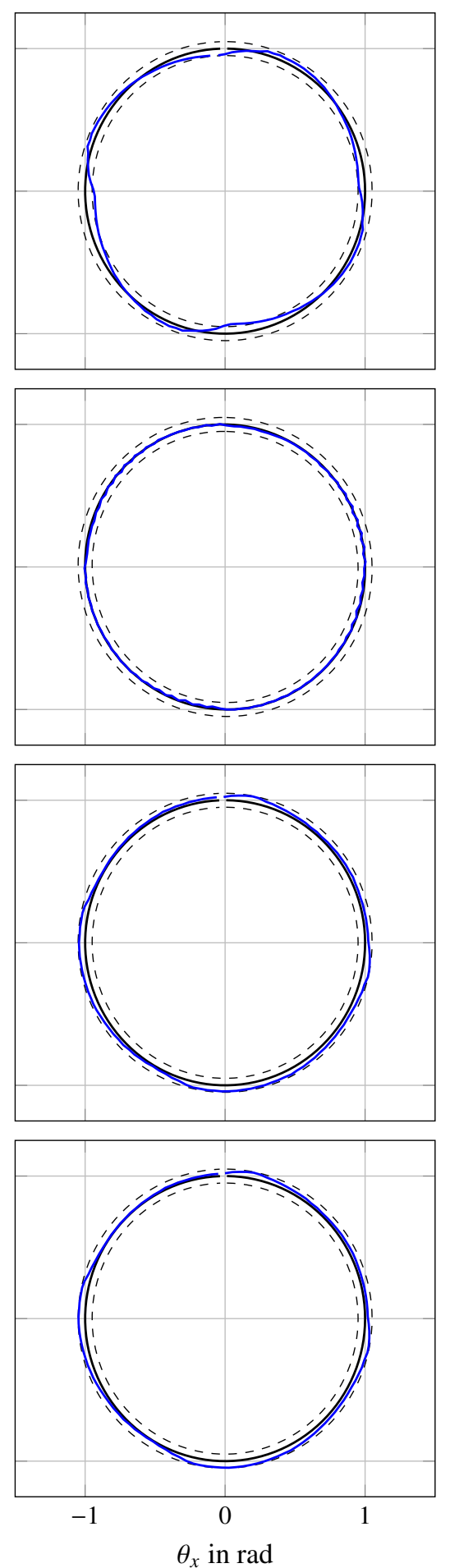

$2 \mathrm{~Hz}$
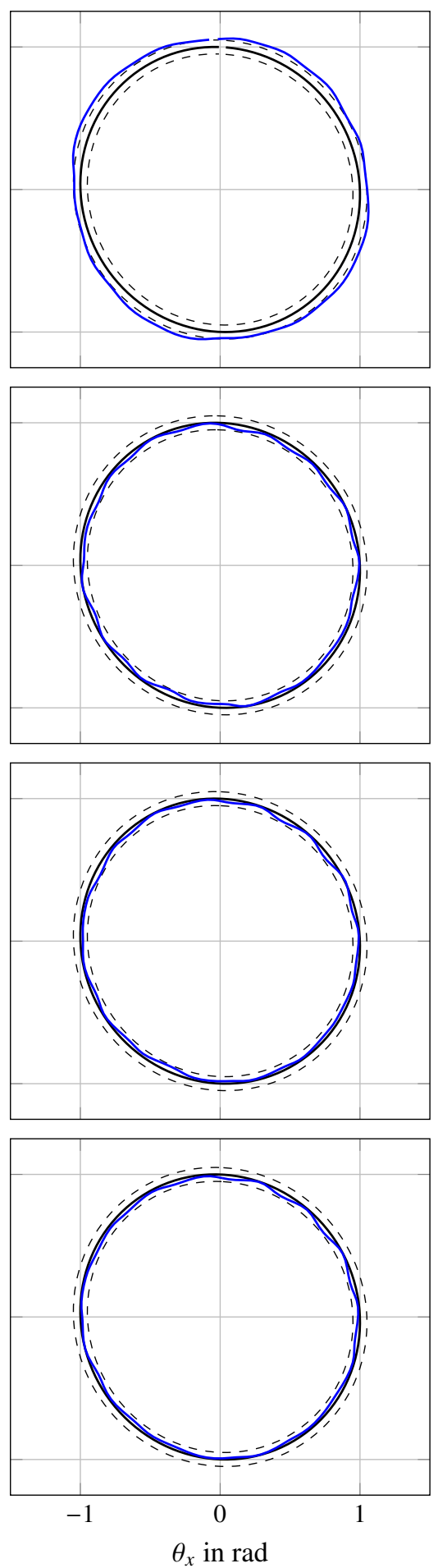

Figure 7: $\theta_{x}-\theta_{y}$ plot for one cycle during Tests 7 (left column), 8 (middle column) and 9 (right column). From top to bottom: P-PI, P-STSMC, P-NAC, P-I\&I-AC. The dashed circles correspond to the $\pm 10^{-2}$ rad accuracy limit introduced in Problem 1. For increased readability, both the error and the accuracy limits have been enlarged by a factor of 5. The largest deviations are observed during the axes' motion reversal, i.e. where the nonlinear nature of friction is more dominant. Under rich excitation (third column) the adaptive controllers adequately suppress the effect of the increased Coulomb friction. 
$0.1 \mathrm{~Hz}$
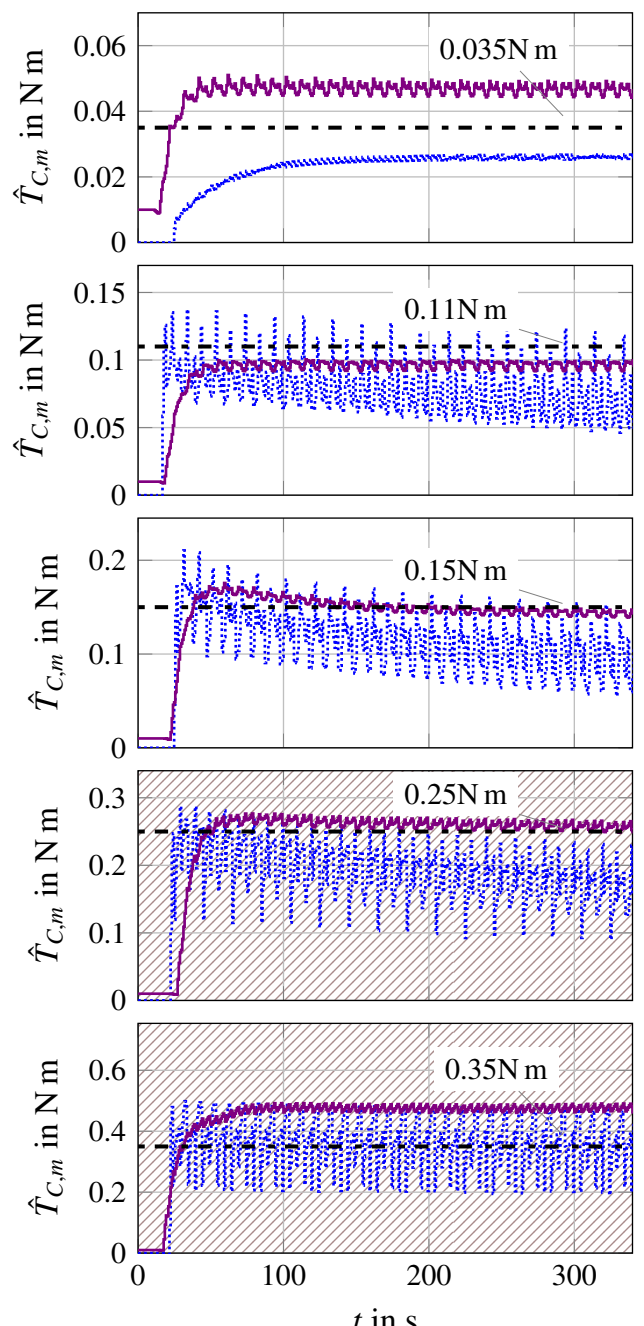

$0.5 \mathrm{~Hz}$
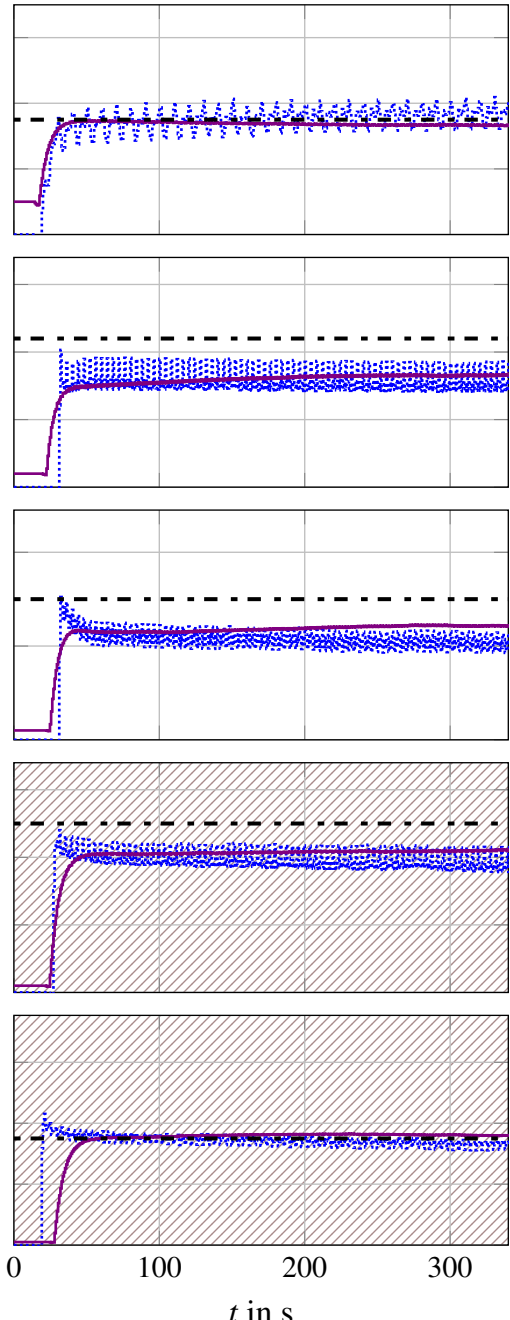

$2 \mathrm{~Hz}$
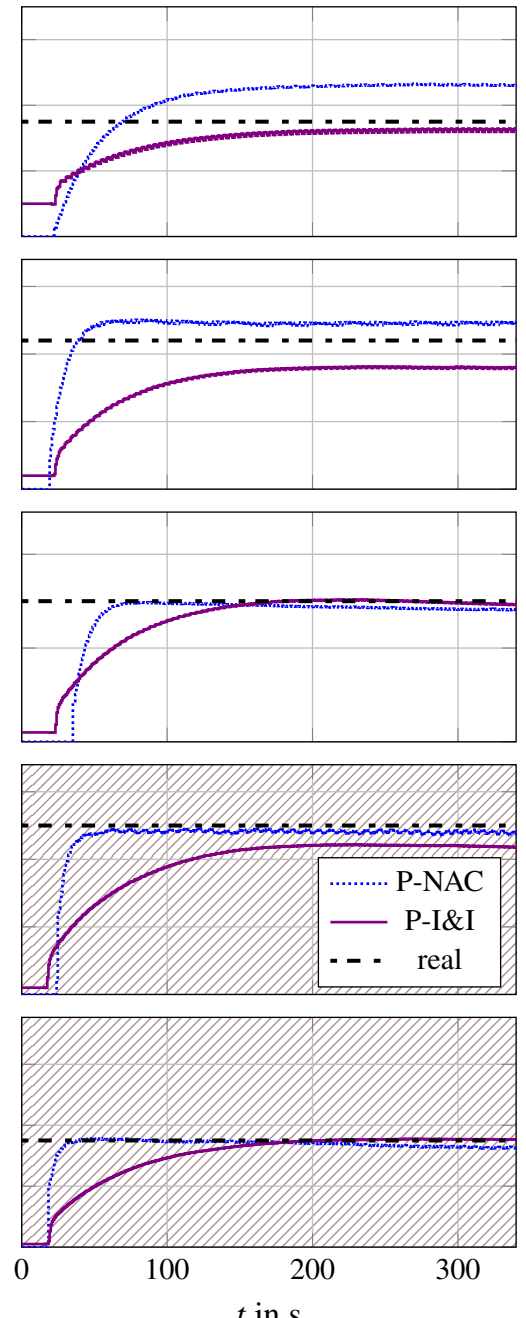

Figure 8: Coulomb friction estimation during all P-NAC and P-I\&I-AC tests. The different cases of friction start with nominal friction and increase from top to bottom. The plots in the stripped area correspond to the extreme friction cases. Since the performance of the adaptive controllers is worse for the case of the slowest reference frequency due to poorer excitation, the effect of nonlinear friction shows up through the tracking error in the estimated parameters' signals (peaks at the instants of the axes' motion reversals). 

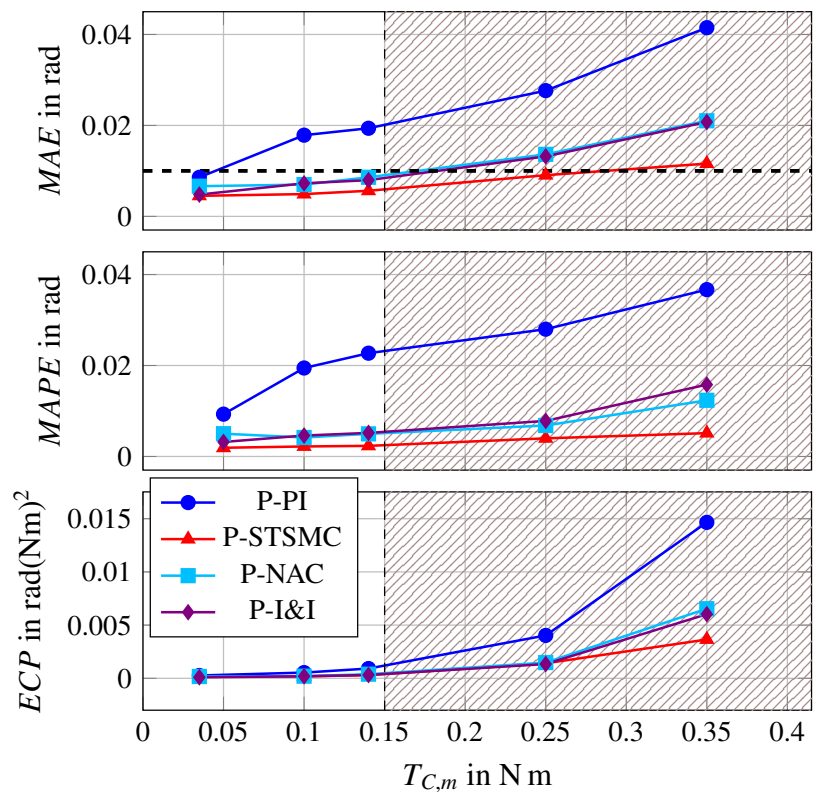

Figure 9: Average MAE (top), MAPE (middle) and ECP (bottom) for all friction cases. The dashed line correspond to the $\pm 10^{-2} \mathrm{rad}$ accuracy bound introduced in Problem 1 and the stripped area denotes the extreme friction cases. It can be seen that the PI degradation causes both deviation errors and a substantial lag between reference and actual position.

be provided in the form of Circular Interpolation Test (CIT) (or ballbar test) plots [85], which illustrate the accuracy of the machine end-tool following a circle of radius 1 . Deviations from this circular path are indicative of the controllers' accuracy with respect to radial or lag errors. Figure 7 shows the CIT of the compared control schemes for the third friction value (Tests 7 9). Here the positioning errors and the accuracy limits have been scaled up by a factor of 5 to improve readability. It can be seen from these graphs that the P-STSMC performs consistently well at all frequencies, while the adaptive controllers only perform better at $2 \mathrm{~Hz}$.

The estimation of the Coulomb friction in all tests for both P-NAC and P-I\&I-AC is shown in Figure 8. It can be seen that in the cases that correspond to larger values of friction or faster reference signals, the estimates are closer to the real parameter value. This is associated to better identifiability of the Coulomb friction and richer excitation, respectively. For P-I\&I-AC, the adaptation of parameter $b$, which captures small perturbations due to shaft uncertainties, also contributes to any deviations of the estimated Coulomb friction from the real value. The overall performance of the four controllers in terms of both accuracy and efficiency is illustrated in Figure 9, where the average

Table 6: Number of tunable parameters and signals required for each controller.

\begin{tabular}{ccccc} 
& $N_{p}$ & Total & $N_{s}$ & Total \\
\hline P-PI & $k_{\text {pos }}, k_{p}, T_{n}$ & 3 & $\theta_{r}, \dot{\theta}_{r}, \theta_{l}, \omega_{m}$ & 4 \\
P-STSMC & $k_{p o s}, k_{1}, k_{2}$ & 3 & $\theta_{r}, \dot{\theta}_{r}, \ddot{\theta}_{r}, \theta_{l}, \omega_{m}, \omega_{l}$ & 6 \\
P-NAC & $k_{p o s}, k, \boldsymbol{\Gamma}, \hat{\boldsymbol{\vartheta}}_{\mathbf{0}}$ & 12 & $\theta_{r}, \dot{\theta}_{r}, \ddot{\theta}_{r}, \boldsymbol{x}$ & 7 \\
P-I\&I-AC & $k_{p o s}, k_{I I}, \boldsymbol{\Gamma}_{\boldsymbol{I I}}, \hat{\boldsymbol{\vartheta}}_{\mathbf{0}}$ & 10 & $\theta_{r}, \dot{\theta}_{r}, \ddot{\theta}_{r}, \theta_{l}, \omega_{m}, \omega_{l}$ & 6 \\
\hline
\end{tabular}

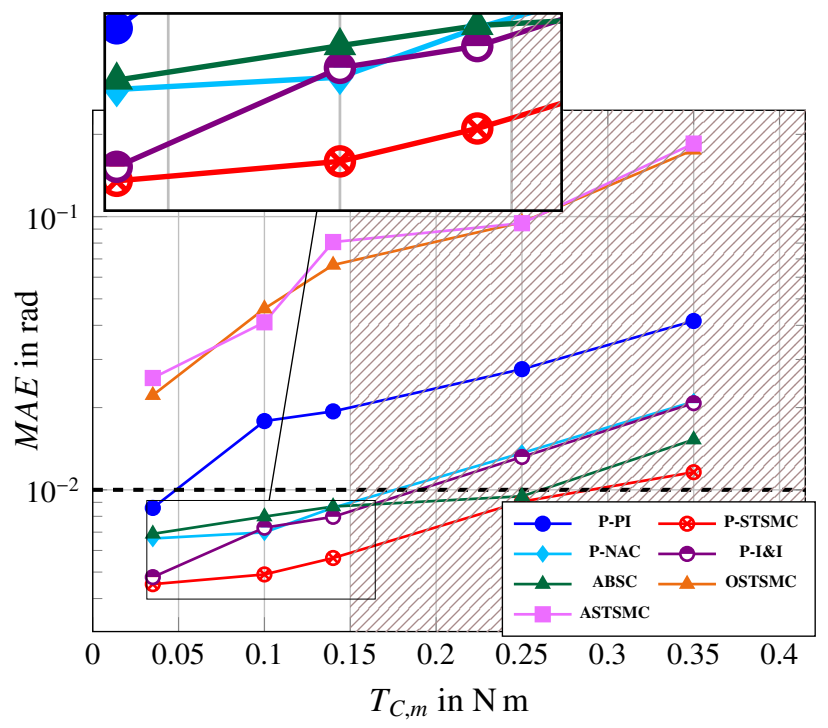

Figure 10: Average MAE for all controllers including the ones presented in [42]. The dashed line corresponds to the $\pm 10^{-2}$ rad accuracy bound and the stripped area denotes the extreme friction cases. The results are presented in logarithmic scale for increased readability. Although the ABSC shows the second best performance in terms of positioning accuracy, its design complexity is significantly higher than any of the proposed cascaded controllers.

MAE, MAPE and ECP are shown for the different friction cases.

Finally, as shown in Table 6, the adaptive controllers have considerably higher design complexity since they involve estimation of unknown parameters, each of which introduces two additional tunable parameters.

\section{Discussion}

Juxtaposing the results presented in this study with the findings documented in [42], reveals that nonlinear control strategies can indeed provide high accuracy axis positioning, robust to unknown and increasing friction. This can be seen in Figure 10 , where the average MAE for each of the 7 considered control methods over the three different reference profiles are plotted against the increasing motor Coulomb friction. The graphs show that four out of the six proposed nonlinear controller clearly outperform the conventional P-PI cascade and maintain the tolerances at least for the non-extreme friction cases.

However, the ranking of the proposed controllers based on the average MAE and the CI over all the experiments, illustrated in Figure 11, shows that of the controllers that outperform the P-PI only one, namely the P-STSMC, has complexity comparable to the conventional cascade. This is very important since high controller complexity implies increased commissioning and maintenance cost.

\section{Conclusions and future work}

The problem of friction-resilient high-accuracy positioning of machine tool axes was addressed in this paper. Three nonlinear position control schemes with cascaded architecture were 

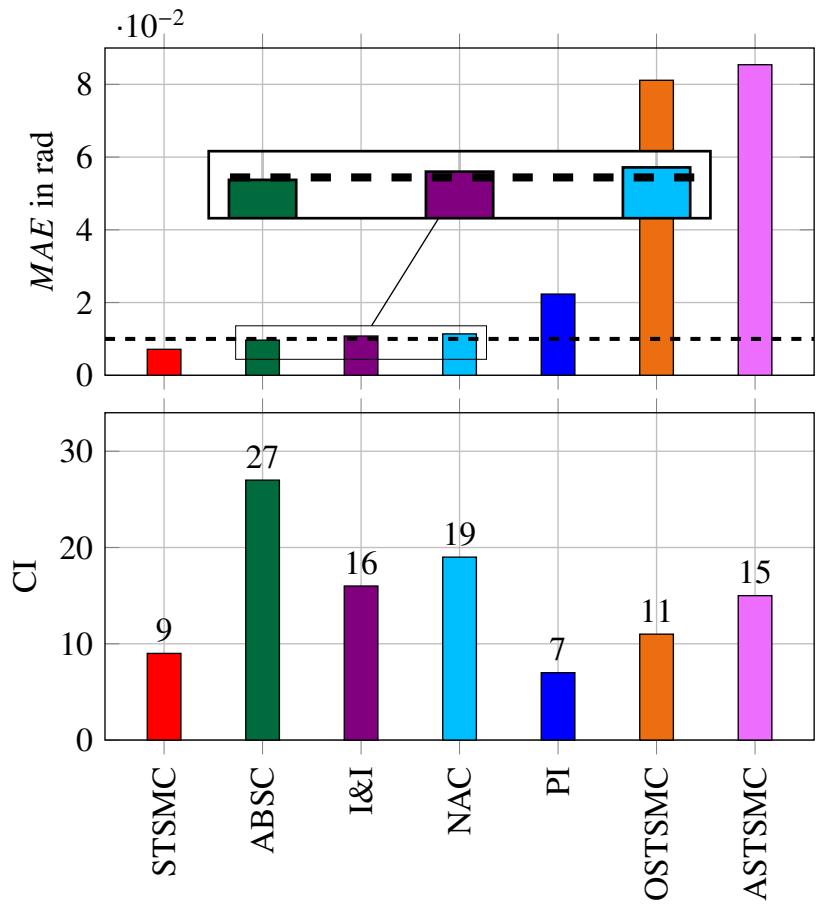

Figure 11: Average MAE (top) and CI (bottom) over all tests for all controllers including the ones presented in [42]. The dashed line correspond to the $\pm 10^{-2} \mathrm{rad}$ accuracy bound introduced in Problem 1 .

designed and implemented on a real single-axis drive-train, consisting of state-of-the-art Siemens equipment. The proposed positioning algorithms were tested under nominal and increasing friction at different reference frequencies and their performance was evaluated based on criteria related to accuracy, robustness and design complexity. Finally, the controllers were compared to the P-PI cascade as well as to the direct-position solutions of [42].

The results demonstrated that the proposed nonlinear solutions outperformed the P-PI cascade, both in nominal operation and under unknown and increased friction. The super-twisting sliding-mode controller exhibited the best average performance over all of the considered cases, with the maximum peak deviation being kept up to 10 times smaller than that of the other controllers. Moreover, its design complexity is directly comparable to the conventional P-PI solution, since it has the same number of tunable parameters. This is a significant advantage over the equally-accurate adaptive backstepping controller, presented in [42].

Future work in this area will focus on the systematic tuning of the P-STSMC scheme based on prescribed workpiece tolerance requirements.

\section{Acknowledgements}

The authors would like to thank Dr. Carsten Hamm and Dr. Hans-Georg Koepken from Siemens AG, Digital Factory Division, Motion Control for their support with integrating the controllers into the existing Siemens software. We would also like to thank them for their invaluable input with respect to the real-life machine tool applications and equipment.
The project was sponsored by Siemens AG.

\section{Appendix A. Proof of ISS property of $e_{\omega}$}

Define the continuously differentiable function $V_{e}\left(e_{\omega}\right)=\frac{1}{2} J_{m} e_{\omega}^{2}$ for which the following inequality holds:

$$
\alpha_{1}\left(\left|e_{\omega}\right|\right) \leq V_{e} \leq \alpha_{2}\left(\left|e_{\omega}\right|\right)
$$

with $\alpha_{1}(y)=\frac{1}{4} y^{2}$ and $\alpha_{2}(y)=y^{2}$ being class $\mathcal{K}_{\infty}$ functions. Taking the time derivative of $V_{e}$ along the trajectories of the error system in (4.37) yields

$$
\begin{aligned}
& \dot{V}_{e}=-k_{I I} e_{\omega}^{2}-e_{\omega} \boldsymbol{\phi}\left(\omega_{m}\right) \boldsymbol{\phi}^{T}\left(\omega_{m}\right) z \leq-k_{I I}\left|e_{\omega}\right|^{2} \\
& +\left|e_{\omega}\right| \cdot\left\|\boldsymbol{\phi}\left(\omega_{m}\right) \boldsymbol{\phi}^{T}\left(\omega_{m}\right)\right\|_{\infty}|z|= \\
& =-k_{I I}(1-\lambda)\left|e_{\omega}\right|^{2}-\left|e_{\omega}\right|\left(k_{I I} \lambda\left|e_{\omega}\right|-\left\|\boldsymbol{\phi}\left(\omega_{m}\right) \boldsymbol{\phi}^{T}\left(\omega_{m}\right)\right\|_{\infty}|z|\right) \\
& \leq-k_{I I}(1-\lambda)\left|e_{\omega}\right|^{2} \triangleq \alpha_{4}\left(\left|e_{\omega}\right|\right), \\
& \forall\left|e_{\omega}\right| \geq \frac{\left\|\phi\left(\omega_{m}\right) \boldsymbol{\phi}^{T}\left(\omega_{m}\right)\right\|_{\infty}}{k_{I I} \lambda}|z| \triangleq \alpha_{3}(|z|)
\end{aligned}
$$

where $0<\lambda<1, \alpha_{4}(\cdot)$ is a class $\mathcal{K}_{\infty}$ function and $\alpha_{3}(\cdot)$ is positive definite in $\mathbb{R}$. Moreover, the existence of the infinity norm of $\phi\left(\omega_{m}\right) \phi^{T}\left(\omega_{m}\right)$ is guaranteed by the boundedness of $\phi\left(\omega_{m}(t)\right), \forall t \geq 0$. Then according to Theorem 4.19 in [86, p. 176] the system in (4.37) is ISS with respect to the input $z$.

[1] H. Gross, J. Hamann, G. Wiegärtner, Electrical feed drives in automation: basics, computation, dimensioning, Publicis MCD Corporate Pub., 2001.

[2] Y. Koren, Control of machine tools, Transactions of the ASME, Journal of Manufacturing Science and Engineering 119 (4) (1997) 749-755.

[3] Y. Koren, Cross-coupled biaxial computer control for manufacturing systems, Journal of Dynamic Systems, Measurement, and Control 102 (4) (1980) 265-272.

[4] G. T. C. Chiu, M. Tomizuka, Contouring control of machine tool feed drive systems: a task coordinate frame approach, IEEE Transactions on Control Systems Technology 9 (1) (2001) 130-139. doi : 10.1109/87. 896754.

[5] L. Settineri, P. Stavropoulos, D. Chantzis, C. Doukas, A. Papacharalampopoulos, G. Chryssolouris, Monitoring and control of manufacturing processes: A review, Procedia CIRP 8 (2013) $421-425$.

[6] N. van de Wouw, H. Pastink, M. F. Heertjes, A. V. Pavlov, H. Nijmeijer, Performance of convergence-based variable-gain control of optical storage drives, Automatica 44 (1) (2008) 15-27.

[7] B. Hunnekens, N. van de Wouw, H. Nijmeijer, Variable gain motion control for transient performance improvement, in: American Control Conference (ACC), 2012, IEEE, 2012, pp. 2467-2472.

[8] B. Hunnekens, N. van de Wouw, M. Heertjes, H. Nijmeijer, Synthesis of variable gain integral controllers for linear motion systems, IEEE Transactions on Control Systems Technology 23 (1) (2015) 139-149.

[9] B. Armstrong-Hlouvry, P. Dupont, C. C. D. Wit, A survey of models, analysis tools and compensation methods for the control of machines with friction, Automatica 30 (7) (1994) 1083 - 1138. doi:10.1016/ 0005-1098 (94) 90209-7.

[10] B. Bona, M. Indri, Friction compensation in robotics: an overview, in: Decision and Control, 2005 and 2005 European Control Conference. CDC-ECC'05. 44th IEEE Conference on, IEEE, 2005, pp. 4360-4367.

[11] R. Konowrocki, T. Szolc, A. Pochanke, A. Prȩgowska, An influence of the stepping motor control and friction models on precise positioning of the complex mechanical system, Mechanical Systems and Signal Processing 70 (2016) 397-413.

[12] G. Liu, A. Goldenberg, Y. Zhang, Precise slow motion control of a directdrive robot arm with velocity estimation and friction compensation, Mechatronics 14 (7) (2004) 821-834. 
[13] L. Marton, B. Lantos, Modeling, identification, and compensation of stick-slip friction, IEEE Transactions on Industrial Electronics 54 (1) (2007) 511-521.

[14] J. Swevers, F. Al-Bender, C. G. Ganseman, T. Projogo, An integrated friction model structure with improved presliding behavior for accurate friction compensation, IEEE Transactions on automatic control 45 (4) (2000) 675-686.

[15] W.-S. Huang, C.-W. Liu, P.-L. Hsu, S.-S. Yeh, Precision control and compensation of servomotors and machine tools via the disturbance observer, IEEE Transactions on Industrial Electronics 57 (1) (2010) 420-429.

[16] Z. Jamaludin, H. Van Brussel, J. Swevers, Quadrant glitch compensation using friction model-based feedforward and an inverse-model-based disturbance observer, in: Advanced Motion Control, 2008. AMC'08. 10th IEEE International Workshop on, IEEE, 2008, pp. 212-217.

[17] Z. Jamaludin, H. Van Brussel, J. Swevers, Friction compensation of an $x y$ feed table using friction-model-based feedforward and an inverse-modelbased disturbance observer, IEEE Transactions on Industrial Electronics 56 (10) (2009) 3848-3853.

[18] V. Lampaert, J. Swevers, F. Al-Bender, Comparison of model and nonmodel based friction compensation techniques in the neighbourhood of pre-sliding friction, in: American Control Conference, 2004. Proceedings of the 2004, Vol. 2, IEEE, 2004, pp. 1121-1126.

[19] M. Ruderman, Tracking control of motor drives using feedforward friction observer, IEEE Transactions on Industrial Electronics 61 (7) (2014) 3727-3735.

[20] M. Ruderman, T. Bertram, Feed-forward friction observer (fffo) for highdynamic motion control, in: Control \& Automation (MED), 2012 20th Mediterranean Conference on, IEEE, 2012, pp. 1019-1024.

[21] M. Ruderman, M. Iwasaki, Observer of nonlinear friction dynamics for motion control, IEEE Transactions on Industrial Electronics 62 (9) (2015) 5941-5949.

[22] M. Ruderman, M. Iwasaki, Analysis of linear feedback position control in presence of presliding friction, IEEJ Journal of Industry Applications 5 (2) (2016) 61-68.

[23] M. Ruderman, Integral control action in precise positioning systems with friction, IFAC-PapersOnLine 49 (13) (2016) 82-86.

[24] D. Rijlaarsdam, P. Nuij, J. Schoukens, M. Steinbuch, Frequency domain based nonlinear feed forward control design for friction compensation, Mechanical Systems and Signal Processing 27 (2012) 551-562.

[25] Y. Ye, C.-B. Yin, Y. Gong, J.-j. Zhou, Position control of nonlinear hydraulic system using an improved pso based pid controller, Mechanical Systems and Signal Processing 83 (2017) 241-259.

[26] C. C. De Wit, P. Lischinsky, Adaptive friction compensation with partially known dynamic friction model, International journal of adaptive control and signal processing 11 (1) (1997) 65-80.

[27] M. Indri, I. Lazzero, A. Antoniazza, A. M. Bottero, Friction modeling and identification for industrial manipulators, in: Emerging Technologies \& Factory Automation (ETFA), 2013 IEEE 18th Conference on, IEEE, 2013, pp. $1-8$.

[28] V. I. Utkin, Sliding mode control design principles and applications to electric drives, IEEE transactions on industrial electronics 40 (1) (1993) 23-36.

[29] V. Utkin, J. Guldner, J. Shi, Sliding mode control in electro-mechanical systems, Vol. 34, CRC press, 2009.

[30] M. Jin, J. Lee, P. H. Chang, C. Choi, Practical nonsingular terminal sliding-mode control of robot manipulators for high-accuracy tracking control, IEEE Transactions on Industrial Electronics 56 (9) (2009) 35933601.

[31] Y. Altintas, K. Erkorkmaz, W.-H. Zhu, Sliding mode controller design for high speed feed drives, CIRP Annals-Manufacturing Technology 49 (1) (2000) 265-270.

[32] L. Dong, W. C. Tang, Adaptive backstepping sliding mode control of flexible ball screw drives with time-varying parametric uncertainties and disturbances, ISA transactions 53 (1) (2014) 110-116.

[33] S. Kang, H. Yan, L. Dong, C. Li, Finite-time adaptive sliding mode force control for electro-hydraulic load simulator based on improved gms friction model, Mechanical Systems and Signal Processing 102 (2018) $117-138$.

[34] K. Khorasani, Adaptive control of flexible-joint robots, IEEE Transactions on Robotics and Automation 8 (2) (1992) 250-267. doi : 10.1109/ 70.134278.

[35] Y. Tan, I. Kanellakopoulos, Adaptive nonlinear friction compensation with parametric uncertainties, in: American Control Conference, 1999. Proceedings of the 1999, Vol. 4, IEEE, 1999, pp. 2511-2515.

[36] L. R. Ray, A. Ramasubramanian, J. Townsend, Adaptive friction compensation using extended kalmanan-bucy filter friction estimation, Control Engineering Practice 9 (2) (2001) 169 - 179. doi:10.1016/ S0967-0661(00) 00104-0.

[37] J. Zhou, Y. Wang, Adaptive backstepping speed controller design for a permanent magnet synchronous motor, Electric Power Applications, IEE Proceedings- 149 (2) (2002) 165-172. doi:10.1049/ip-epa: 20020187.

[38] D. Garagić, K. Srinivasan, Adaptive friction compensation for precision machine tool drive, Control Engineering Practice 12 (11) (2004) 14511464, cited By 0. doi : 10.1016/j . conengprac.2003.10.006.

[39] Y. Zhang, X. Ren, Adaptive backstepping control of dual-motor driving servo systems with friction, Proceedings - 2014 6th International Conference on Intelligent Human-Machine Systems and Cybernetics, IHMSC 20141 (1) (2014) 214-217. doi:10.1109/IHMSC . 2014.60.

[40] S. Avila-Becerril, A. Loría, E. Panteley, Global position-feedback tracking control of flexible-joint robots, in: American Control Conference (ACC), 2016, IEEE, 2016, pp. 3008-3013.

[41] Y. Wang, D. Wang, T. Chai, Modeling and control compensation of nonlinear friction using adaptive fuzzy systems, Mechanical Systems and Signal Processing 23 (8) (2009) 2445-2457.

[42] D. Papageorgiou, M. Blanke, H. H. Niemann, J. H. Richter, Frictionresilient position control for machine toolsadaptive and sliding-mode methods compared, Control Engineering Practice 75 (2018) 69-85.

[43] G. Straffelini, Friction and Wear: Methodologies for Design and Control (Springer Tracts in Mechanical Engineering), 1st Edition, Springer, 2015.

[44] T. M. Jahns, Torque production in permanent-magnet synchronous motor drives with rectangular current excitation, IEEE Transactions on Industry Applications 4 (1984) 803-813.

[45] H. Bolton, R. Ashen, Influence of motor design and feed-current waveform on torque ripple in brushless dc drives, in: IEEE Proceedings B-Electric Power Applications, Vol. 131, IET, 1984, pp. 82-90.

[46] M. Dai, A. Keyhani, T. Sebastian, Torque ripple analysis of a pm brushless dc motor using finite element method, IEEE Transactions on energy conversion 19 (1) (2004) 40-45.

[47] A. Tripathi, A. M. Khambadkone, S. K. Panda, Torque ripple analysis and dynamic performance of a space vector modulation based control method for ac-drives, IEEE transactions on power electronics 20 (2) (2005) 485492.

[48] A. Gebregergis, M. H. Chowdhury, M. S. Islam, T. Sebastian, Modeling of permanent-magnet synchronous machine including torque ripple effects, IEEE Transactions on Industry Applications 51 (1) (2015) 232-239.

[49] R. Carlson, A. A. Tavares, J. P. Bastos, M. Lajoie-Mazenc, Torque ripple attenuation in permanent magnet synchronous motors, in: Industry Applications Society Annual Meeting, 1989., Conference Record of the 1989 IEEE, IEEE, 1989, pp. 57-62.

[50] G. Ferretti, G. Magnani, P. Rocco, Modeling, identification, and compensation of pulsating torque in permanent magnet ac motors, IEEE Transactions on Industrial Electronics 45 (6) (1998) 912-920.

[51] O. Egeland, J. T. Gravdahl, Modeling and simulation for automatic control, Vol. 76, Marine Cybernetics Trondheim, Norway, 2002.

[52] K. Johanastrom, C. Canudas-De-Wit, Revisiting the lugre friction model, IEEE control Systems 28 (6) (2008) 101-114.

[53] G. Ferretti, G. Magnani, P. Rocco, An integral friction model, in: Robotics and Automation, 2004. Proceedings. ICRA'04. 2004 IEEE International Conference on, Vol. 2, IEEE, 2004, pp. 1809-1813.

[54] G. Ferretti, G. Magnani, P. Rocco, Single and multistate integral friction models, IEEE Transactions on Automatic Control 49 (12) (2004) 22922297.

[55] C. Rebelein, M. Zaeh, Friction in feed drives of machine tools: investigation, modeling and validation, Production Engineering (2016) 1-11.

[56] M. Ruderman, T. Bertram, Two-state dynamic friction model with elastoplasticity, Mechanical Systems and Signal Processing 39 (1) (2013) 316332.

[57] Y. Wan, T. Dodd, C. Wong, R. Harrison, K. Worden, Kernel based modelling of friction dynamics, Mechanical Systems and Signal Processing 22 (1) (2008) 66-80.

[58] C. C. De Wit, H. Olsson, K. J. Astrom, P. Lischinsky, A new model for control of systems with friction, IEEE Transactions on Automatic Control 
40 (3) (1995) 419-425.

[59] M. Boegli, T. De Laet, J. De Schutter, J. Swevers, A smoothed gms friction model for moving horizon friction state and parameter estimation, in: Advanced Motion Control (AMC), 2012 12th IEEE International Workshop on, IEEE, 2012, pp. 1-6.

[60] M. M. Seron, J. H. Braslavsky, G. C. Goodwin, Fundamental limitations in filtering and control, Springer Science \& Business Media, 2012.

[61] R. H. Middleton, Trade-offs in linear control system design, Automatica 27 (2) (1991) 281-292.

[62] A. Levant, Sliding order and sliding accuracy in sliding mode control, International journal of control 58 (6) (1993) 1247-1263.

[63] A. Levant, Higher-order sliding modes, differentiation and outputfeedback control, International Journal of Control 76 (9-10) (2003) 924941.

[64] V. Utkin, Methods for constructing discontinuity planes in multidimensional variable structure systems, Automation and Remote Control 39 (1978) 1466-1470.

[65] A. Levant, Construction principles of output-feedback 2-sliding mode design, in: Decision and Control, 2002, Proceedings of the 41st IEEE Conference on, Vol. 1, 2002, pp. 317-322 vol.1. doi : 10.1109/CDC. 2002. 1184511.

[66] A. Levant, Principles of 2-sliding mode design, Automatica 43 (4) (2007) 576 - 586. doi: 10.1016/j . automatica.2006.10.008.

[67] G. Bartolini, A. Ferrara, E. Usai, V. I. Utkin, On multi-input chatteringfree second-order sliding mode control, IEEE Transactions on Automatic Control 45 (9) (2000) 1711-1717. doi:10.1109/9.880629.

[68] G. Bartolini, A. Ferrara, E. Usai, Chattering avoidance by second-order sliding mode control, IEEE Transactions on Automatic Control 43 (2) (1998) 241-246. doi :10.1109/9.661074.

[69] Y. B. Shtessel, J. a. Moreno, F. Plestan, L. M. Fridman, A. S. Poznyak, Super-twisting adaptive sliding mode control: A Lyapunov design, 49th IEEE Conference on Decision and Control (CDC) (2010) 51095113doi:10.1109/CDC.2010.5717908.

[70] C. Edwards, Y. B. Shtessel, Adaptive continuous higher order sliding mode control, Automatica 65 (2016) 183-190.

[71] J. Rivera, L. Garcia, C. Mora, J. J. Raygoza, S. Ortega, Super-Twisting Sliding Mode in Motion Control Systems (1993).

[72] C. Baratieri, H. Pinheiro, New variable gain super-twisting sliding mode observer for sensorless vector control of nonsinusoidal back-emf pmsm, Control Engineering Practice 52 (2016) 59-69, cited By 0. doi:10. 1016/j.conengprac. 2016.04.003.

[73] J. a. Moreno, M. Osorio, Strict lyapunov functions for the super-twisting algorithm, IEEE Transactions on Automatic Control 57 (4) (2012) 10351040. doi:10.1109/TAC. 2012.2186179.

[74] A. Chalanga, F. Plestan, Finite time stabilization of an uncertain chain of integrators by integral sliding mode approach, IFAC-PapersOnLine 50 (1) (2017) 9613-9618.

[75] K. J. Åstrom, Adaptive control (Addison-Wesley series in electrical and computer engineering), Addison-Wesley, 1989.

[76] P. A. Ioannou, J. Sun, Robust adaptive control, Vol. 1, PTR Prentice-Hall Upper Saddle River, NJ, 1996.

[77] M. Krstic, I. Kanellakopoulos, P. V. Kokotovic, Nonlinear and Adaptive Control Design, 1st Edition, Wiley-Interscience, 1995.

[78] A. Astolfi, R. Ortega, Immersion and invariance: a new tool for stabilization and adaptive control of nonlinear systems, IEEE Transactions on Automatic Control 48 (4) (2003) 590-606. doi:10.1109/TAC.2003. 809820.

[79] A. Astolfi, D. Karagiannis, R. Ortega, Nonlinear and Adaptive Control with Applications (Communications and Control Engineering), 2008th Edition, Springer, 2008.

[80] A. Donaire, T. Perez, Y. R. Teo, Robust speed tracking control of synchronous motors using immersion and invariance, in: 2012 7th IEEE Conference on Industrial Electronics and Applications (ICIEA), 2012, pp. 1482-1487. doi:10.1109/ICIEA . 2012.6360958.

[81] T. Wimböck, C. Ott, G. Hirzinger, Immersion and invariance control for an antagonistic joint with nonlinear mechanical stiffness, in: 49th IEEE Conference on Decision and Control (CDC), 2010, pp. 1128-1135. doi : 10.1109/CDC. 2010.5717891.

[82] M.-F. Danca, On the uniqueness of solutions to a class of discontinuous dynamical systems, Nonlinear Analysis: Real World Applications 11 (3) (2010) 1402-1412.

[83] A. F. Filippov, Differential equations with discontinuous right-hand side, Matematicheskii sbornik 93 (1) (1960) 99-128.

[84] H. Dyratok, Huco Vari-tork adjustable-friction clutches, http://www . huco.com/products . asp? p=true\&cat=155 [Accessed: January 27] (2017).

[85] G. T. Smith, Machine Tool Metrology: An Industrial Handbook, Springer, 2016.

[86] H. Khalil, Nonlinear Systems, Prentice Hall, 2002. 